\title{
Four tyrosine residues of the rice immune receptor XA21 are not required for interaction with the co-receptor OsSERK2 or resistance to Xanthomonas oryzae pv. oryzae
}

\author{
Daniel F Caddell ${ }^{1,2}$ ， Tong Wei ${ }^{1,3}$ ，Sweta Sharma ${ }^{1}$, Man-Ho Oh ${ }^{4,5}$, Chang-Jin Park ${ }^{1,6}$, Patrick Canlas ${ }^{1}$, \\ Steven C Huber ${ }^{4,7}$, Pamela C Ronald ${ }^{\text {Corresp. } 1}$ \\ 1 Department of Plant Biology and the Genome Center, University of California, Davis, Davis, CA, United States \\ 2 Current affiliation: Department of Plant and Microbial Biology, University of California, Berkeley, Berkeley, California (CA), United States \\ 3 Current affiliation: China National GeneBank, BGI Research, Shenzen, China \\ 4 Department of Plant Biology, University of Illinois at Urbana-Champaign, Urbana, IL, United States \\ 5 Current affiliation: Department of Biological Science, Chungnam National University, Daejeon, South Korea \\ 6 Current affiliation: Department of Bioresources and Engineering and PERI, Sejong University, Seoul, South Korea \\ 7 Agricultural Research Service, United States Department of Agriculture, Urbana, IL, USA \\ Corresponding Author: Pamela C Ronald \\ Email address: pcronald@ucdavis.edu
}

Tyrosine phosphorylation has emerged as an important regulator of plasma membrane localized immune receptors activity. Here we investigate the role of tyrosine phosphorylation in the regulation of rice XA21-mediated immunity. We demonstrate that the juxtamembrane and kinase domain of Escherichia coli-expressed XA21 (XA21JK) autophosphorylates on tyrosine residues. Directed mutagenesis of four out of the nine tyrosine residues in XA21JK reduced autophosphorylation. These sites include Tyr ${ }^{698}$ in the juxtamembrane domain, and $\mathrm{Ty}^{786}, \mathrm{Tyr}^{907}$, and $\mathrm{Ty}^{909}$ in the kinase domain. Rice plants expressing XA21-GFP fusion proteins or proteins with these tyrosine residues individually mutated to phenylalanine (XA21 $1^{\mathrm{YF}}-\mathrm{GFP}$ ), which prevents phosphorylation at these sites, maintain resistance to Xanthomonas oryzae pv. oryzae. In contrast, plants expressing phosphomimetic XA21 variants with tyrosine mutated to aspartate (XA21 ${ }^{\mathrm{YD}}$-GFP) were susceptible. In vitro purified XA21JK ${ }^{\Upsilon 698 F}$, XA21JK ${ }^{\text {Y907F }}$, and $X A 21 J \mathrm{~K}^{\mathrm{Y} 909 \mathrm{~F}}$ variants are

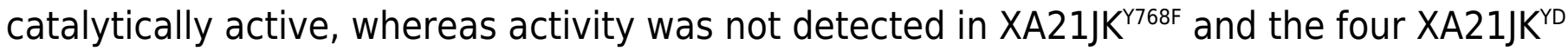
variants. We previously demonstrated that interaction of XA21 with the co-receptor

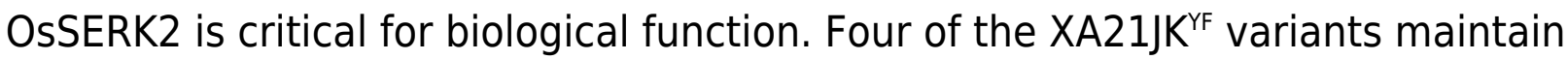
interaction with OsSERK2 as well as the XA21 binding (XB) proteins XB3 and XB15 in yeast, suggesting that these four tyrosine residues are not required for their interaction. Taken together, these results suggest that XA21 is capable of tyrosine autophosphorylation, but the identified tyrosine residues are not required for activation of XA21-mediated immunity or interaction with predicted XA21 signaling proteins. 
1 Four tyrosine residues of the rice immune receptor XA21 are not required for

2 interaction with the co-receptor OSSERK2 or resistance to Xanthomonas oryzae pv.

3 oryzae

4 Daniel F. Caddell ${ }^{1 \mathrm{a}}$, Tong Wei ${ }^{1 \mathrm{~b}}$, Sweta Sharma ${ }^{1}$, Man-Ho Oh${ }^{2 c}$, Chang-Jin Park ${ }^{1 \mathrm{~d}}$, Patrick

5 Canlas $^{1}$, Steven C. Huber ${ }^{2,3}$, and Pamela C. Ronald ${ }^{1}$

6

$7 \quad{ }^{1}$ Department of Plant Pathology and the Genome Center, University of California Davis, Davis, 8 CA, USA

$9{ }^{2}$ Department of Plant Biology, University of Illinois, Urbana, IL, USA

$10{ }^{3}$ United States Department of Agriculture, Agricultural Research Service, Urbana, IL, USA

11 aCurrent address: Department of Plant and Microbial Biology, University of California,

12 Berkeley, Berkeley, CA, USA

$13{ }^{\mathrm{b} C u r r e n t}$ address: BGI Research, China National GeneBank, Jinsha Road, Dapeng District,

14 Shenzhen 518116, China

$15{ }^{\mathrm{c} C u r r e n t}$ address: Department of Biological Science, College of Biological Sciences and

16 Biotechnology, Chungnam National University, Daejeon, South Korea

17 dCurrent address: Department of Bioresources Engineering and PERI, Sejong University, Seoul,

18 South Korea

$20 *$ To whom correspondence should be addressed.

21 E-mail: pcronald@ucdavis.edu

22 Tel: +1 530-752-1654

23 Fax: +1 530-752-6088 


\section{Abstract}

25 Tyrosine phosphorylation has emerged as an important regulator of plasma membrane

26 localized immune receptors activity. Here we investigate the role of tyrosine phosphorylation in

27 the regulation of rice XA21-mediated immunity. We demonstrate that the juxtamembrane and

28 kinase domain of Escherichia coli-expressed XA21 (XA21JK) autophosphorylates on tyrosine

29 residues. Directed mutagenesis of four out of the nine tyrosine residues in XA21JK reduced

30 autophosphorylation. These sites include $\mathrm{Tyr}^{698}$ in the juxtamembrane domain, and $\mathrm{Tyr}^{786}$,

$31 \mathrm{Tyr}^{907}$, and $\mathrm{Tyr}^{909}$ in the kinase domain. Rice plants expressing XA21-GFP fusion proteins or

32 proteins with these tyrosine residues individually mutated to phenylalanine (XA21 $\left.{ }^{\mathrm{YF}}-\mathrm{GFP}\right)$,

33 which prevents phosphorylation at these sites, maintain resistance to Xanthomonas oryzae pv.

34 oryzae. In contrast, plants expressing phosphomimetic XA21 variants with tyrosine mutated to

35 aspartate (XA21 ${ }^{\mathrm{YD}}$-GFP) were susceptible. In vitro purified XA21JK ${ }^{\mathrm{Y} 698 \mathrm{~F}}$, XA21JK ${ }^{\mathrm{Y} 907 \mathrm{~F}}$, and

$36 \mathrm{XA} 1 \mathrm{JK}^{\mathrm{Y} 909 \mathrm{~F}}$ variants are catalytically active, whereas activity was not detected in $\mathrm{XA} 21 \mathrm{JK}^{\mathrm{Y} 768 \mathrm{~F}}$

37 and the four XA21JK ${ }^{\mathrm{YD}}$ variants. We previously demonstrated that interaction of XA21 with the

38 co-receptor OsSERK2 is critical for biological function. Four of the $\mathrm{XA} 21 \mathrm{JK}^{\mathrm{YF}}$ variants maintain

39 interaction with OsSERK2 as well as the XA21 binding (XB) proteins XB3 and XB15 in yeast,

40 suggesting that these four tyrosine residues are not required for their interaction. Taken together,

41 these results suggest that XA21 is capable of tyrosine autophosphorylation, but the identified

42 tyrosine residues are not required for activation of XA21-mediated immunity or interaction with

43 predicted XA21 signaling proteins.

44

45 


\section{Introduction}

48

49

50

51

52

53

54

55

56

57

Plasma membrane-localized immune receptors initiate plant defense responses upon recognition of conserved microbial molecules. Three well-characterized plasma membranelocalized immune receptors are Arabidopsis FLAGELLIN-SENSING 2 (FLS2), Arabidopsis EFTu RECEPTOR (EFR), and rice XANTHOMONAS RESISTANCE 21 (XA21). FLS2, EFR, and XA21 recognize bacterial flagellin (Gomez-Gomez \& Boller 2000), bacterial elongation factorTu (Zipfel et al. 2006), and the Xanthomonas oryzae pv. oryzae (Xoo) peptide Required for activation of $\underline{X A} 21-m e d i a t e d$ immunity $\underline{X}(\operatorname{RaxX})$ (Pruitt et al. 2015), respectively. Immune activation of FLS2, EFR, and XA21 requires interaction with members of the SOMATIC EMBRYOGENESIS RECEPTOR-LIKE KINASE (SERK) family of proteins. In Arabidopsis, EFR and FLS2 interact with AtSERK3, commonly referred to as BRI1-ASSOCIATED KINASE1 (BAK1) (Chinchilla et al. 2007). Recognition of the cognate bacterial peptide induces direct heterodimerization and transphosphorylation between BAK1 and its partner receptor (Chinchilla et al. 2007). In rice, XA21 and the immune receptors OsFLS2 (Takai et al. 2008) and XA3 (Xiang et al. 2006) require OsSERK2 for biological function (Chen et al. 2014). The interaction and transphosphorylation between XA21 and OsSERK2 occurs in the absence of Xoo (Chen et al. 2014). interactions with XA21 BINDING PROTEINS (XBs). For example, XB3, an E3-ubiquitin ligase, is activated by XA21 and is predicted to assist in XA21-mediated immunity through ubiquitination of proteins (Wang et al. 2006). XB15, a protein phosphatase, dephosphorylates XA21 residues to attenuate XA21-mediated immunity (Park et al. 2008). XB24, an ATPase, maintains XA21 in an inactive state by promoting autophosphorylation of XA21 residues. 
70 Bacterial recognition promotes disassociation of XB24 from XA21, leading to kinase activation

71 (Chen et al. 2010b). Although specific phosphosites are presumed to be involved that activate or

72 inhibit XA21 kinase activity, they are largely unknown. XA21 kinase activity is required for full

73 functionality. Rice plants expressing a catalytically inactive XA21 carrying a glutamate mutation

74 in the conserved lysine residue of the XA21 catalytic domain (XA2 $1^{\mathrm{K} 736 \mathrm{E}}$ ) are partially resistant

75 to Xoo (Andaya \& Ronald 2003). Another XA21 variant, which has the aspartic acid residue

76 required for phospho-transfer (Nolen et al. 2004) swapped to asparagine (XA21 $\left.{ }^{\mathrm{D} 842 \mathrm{~N}}\right)$, is also

77 catalytically inactive (Chen et al. 2014). Phosphorylation of serine and threonine residues in the

78 juxtamembrane (JM) domain of XA21 regulate protein stability (Xu et al. 2006), kinase activity

79 (Chen et al. 2010a), and protein-protein interactions (Chen et al. 2010a; Park et al. 2008).

80 While serine and threonine are the major sites of phosphorylation in most plant proteins

81 (Nakagami et al. 2010), tyrosine is also an important residue for regulating plant growth,

82 development, and immunity (Macho et al. 2015). To study tyrosine phosphorylation, tyrosine

83 residues can be modified using site-directed mutagenesis to phenylalanine ( $\mathrm{Y}$-to-F, or ' $\mathrm{YF}$ ' in

84 this paper) or aspartate (Y-to-D, or 'YD' in this paper), which prevents phosphorylation or serves

85 to mimic the negative charge of a phosphorylated tyrosine (phosphomimetic), respectively. For

86 example, the Arabidopsis leucine-rich repeat receptor-like kinase (LRR-RLK)

87 BRASSINOSTEROID INSENSITIVE 1 (BRI1), which recognizes the growth hormone

88 brassinosteroid, is phosphorylated on $\mathrm{Tyr}^{831}$ and $\mathrm{Tyr}^{956}$ in planta. BRI1 variants that carry $\mathrm{Tyr}^{831}$

89 replaced with phenylalanine $\left(\mathrm{BRI} 1^{\mathrm{Y} 831 \mathrm{~F}}\right)$ retain kinase activity but display increased overall

90 growth with altered leaf shape and reduced flowering time when expressed in the bril-5 mutant

91 background (Oh et al. 2009). In contrast, the BRI1 variant with $\mathrm{Tyr}^{956}$ modified to phenylalanine

$92\left(\mathrm{BRI}^{\mathrm{Y} 956 \mathrm{~F}}\right)$ is catalytically inactive (Oh et al. 2009). Phosphorylation of Tyr ${ }^{956}$ is inhibitory 
93 rather than essential for activity (Oh et al. 2012), consistent with results obtained with the

94 Arachis Symbiosis Receptor Kinase, AhSYMRK (Paul et al. 2014), where autophosphorylation

95 of the homologous tyrosine residue on AhSYMRK $\left(\mathrm{Tyr}^{670}\right)$ also inhibited catalytic activity.

96 Collectively, these results suggest that tyrosine phosphorylation is critical for BRI1-mediated

97 growth and development.

98 Tyrosine phosphorylation also serves an important role in EFR-mediated immunity.

99 Phosphorylation of $\mathrm{Tyr}^{836}$ is necessary for activating EFR-mediated immunity. EFR ${ }^{\mathrm{Y} 836 \mathrm{~F}}$ mutants

100 are capable of elf18-induced dimerization with BAK1, but fail to initiate an EFR-mediated

101 immune response. As a means to incapacitate EFR-mediated immunity, phytopathogenic

102 Pseudomonas syringae secretes a tyrosine phosphatase, HopAO1, that dephosphorylates

103 phospho-Tyr ${ }^{836}$ (Macho et al. 2014). The role of tyrosine phosphorylation was also assessed in

104 the Arabidopsis LysM-RLK CHITIN ELICITOR RECEPTOR KINASE 1 (CERK1). Similar to

105 EFR, autophosphorylation of the analogous tyrosine residue, $\mathrm{Tyr}^{428}$, is required for in vivo

106 activation (Suzuki et al. 2018) and chitin-triggered CERK1 activation (Liu et al. 2018).

107 Dephosphorylation of $\mathrm{Tyr}^{428}$ by the CERK1-interacting protein phosphatase 1 (CIPP1) reduces

108 CERK1 signaling (Liu et al. 2018).

109

While the significance of tyrosine phosphorylation regulating LRR-RLKs in Arabidopsis

110 and Arachis has been demonstrated for some proteins, its role has not been studied in other

111 species. In this study, we investigated the role of tyrosine phosphorylation of rice XA21. We

112 found that Escherichia coli-expressed XA21 autophosphorylates on tyrosine residues, and that

$113 \mathrm{Tyr}^{698}, \mathrm{Tyr}^{786}, \mathrm{Tyr}^{907}$, and $\mathrm{Tyr}^{909}$ are potential autophosphorylation sites. However, transgenic

114 rice expressing XA21 variants with these tyrosine residues replaced with phenylalanine

$115\left(\mathrm{XA} 21^{\mathrm{YF}}\right)$ maintain resistance to $\mathrm{Xoo}$ and interaction with $\mathrm{XBs}$, suggesting that XA21 is capable 
116 of tyrosine autophosphorylation, but the identified tyrosine residues are not required for

117 activation of XA21-mediated immunity or interaction with predicted XA21 signaling proteins.

118

119 Materials and methods

120 Site-directed mutagenesis and plasmid construction

121 A previously generated GST-XA21JK construct (Liu et al. 2002) was used as the template for

122 site-directed mutagenesis using the QuikChange XL Site-Directed Mutagenesis Kit (Stratagene).

123 Individual constructs were generated with the following substitutions: Y698F, Y723F, Y786F,

124 Y829F, Y889F, Y894F, Y907F, Y909F, and Y936F. GST-XA21JK variants K736E, S697A,

125 S697D, T705A, T705E, T680A, S699A were generated previously (Chen et al. 2010a).

$126 \mathrm{XA} 21 \mathrm{JK} / \mathrm{pENTR}$ (Chen et al. 2014) was used as the template for site-directed mutagenesis of

127 Y698F, Y698D, Y786F, Y786D, Y907F, Y907D, Y909F, and Y909D. Gateway cloning using

128 Gateway LR Clonase (Invitrogen) was utilized to recombine XA21JK/pENTR construct and

129 mutant variants into a His-Nus-fusion vector (Chern et al. 2005; Schwessinger et al. 2011) for in

130 vitro kinase autophosphorylation assay. His-Nus-XA21 JK ${ }^{\mathrm{D} 842 \mathrm{~N}}$ was previously generated (Chen

131 et al. 2014). The pNlexA-XA21JK vectors used for yeast analyses were similarly constructed by

132 recombining the XA21JK/pENTR constructs into pNlexAgtwy (a gateway compatible vector

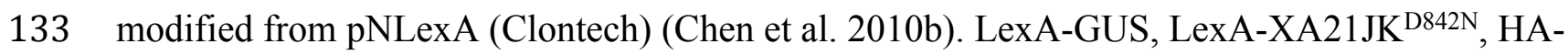

134 XB3, HA-XB15, and HA-OsSERK2JMK vectors were generated previously (Chen et al. 2010a;

135 Chen et al. 2014). XA21-GFP/pC1300 (Park et al. 2010) was used as template for site-directed

136 mutagenesis of Y698F, Y698D, Y786F, Y786D, Y907F, Y907D, Y909F, and Y909D for rice

137 studies. Point mutations in the appropriate vectors were introduced as described above. All 
138 constructs were sequenced in both directions to verify specific mutations and lack of additional

139 mutations.

140

141 Rice transformation

142 Rice transformations were performed as described previously (Chern et al. 2005). Agrobacterium

143 strain EHA105 was used to infect calli generated from the Kitaake rice cultivar. Transformants

144 were selected using hygromycin as a selectable marker and later confirmed by PCR using

145 transgene specific primers (Supplemental Table 1).

146

147 Plant growth conditions and inoculations assays

148 Rice plants (Oryza sativa ssp. japonica L., cultivar Kitaake) were grown in the greenhouse for

149 six weeks and transferred to growth chambers for inoculation with $14 \mathrm{~h}$ day/ $10 \mathrm{~h}$ night cycle,

$15028 / 26^{\circ} \mathrm{C}$ temperature and 85-95\% humidity. Xoo Philippine race 6 strain PXO99Az (PXO99 in

151 this paper), was cultured on PSA media containing cephalexin for 2-3 days and suspended in

152 sterile water to a final $\mathrm{OD}_{600}$ of 0.5 . Healthy and fully expanded leaves of each plant were

153 inoculated using the scissors inoculation method that has been established previously (Chern et

154 al. 2005). Infection was measured 14 days later.

155

156 Protein extraction

157 Approximately $7 \mathrm{~cm}(0.05 \mathrm{~g})$ of tissue from fully expanded rice leaves were crushed to a fine

158 powder in liquid nitrogen. Samples were incubated in rice extraction buffer (2 mM EDTA, 0.15

$159 \mathrm{M} \mathrm{NaCl}, 0.01 \mathrm{M}$ sodium phosphate, $\mathrm{pH}$ 7.2, 1\% (v/v) Triton X-100, $10 \mathrm{mM} ß-\mathrm{Me}, 1 \mathrm{mM}$ PMSF,

$1601 \%(\mathrm{v} / \mathrm{v})$ sigma protease inhibitor cocktail P-9599) for $30 \mathrm{~min}$ on ice. Samples were cleared by 
161 centrifugation at $13,000 \mathrm{rpm}, 4{ }^{\circ} \mathrm{C}$, for $10 \mathrm{~min}$. Protein was denatured in $4 \mathrm{x}$ SDS loading buffer

162 and incubated at $80{ }^{\circ} \mathrm{C}$ for $10 \mathrm{~min}$.

163

164 Gene expression assays

165 Gene expression assays were performed as previously described (Pruitt et al. 2015). Rice plants

166 were grown hydroponically and watered twice a week with Hoagland solution. About $2 \mathrm{~cm}$ leaf

167 strips from 4-week-old plants were floated in sterile water overnight. The leaf tissues were

168 treated for $6 \mathrm{~h}$ in the second morning with water, $1 \mu \mathrm{M}$ nonsulfated or sulfated 21 -amino acid

169 RaxX peptides (RaxX21-Y or RaxX21-sY, respectively; Pacific Immunology, Ramona, CA,

170 USA), and immediately frozen in liquid nitrogen for RNA extraction. Total RNA was isolated

171 with TRIzol reagent (Invitrogen, Carlsbad, CA, USA), treated with DNase I, and used for cDNA

172 synthesis with Multiscribe Reverse Transcriptase (Thermo Fisher Scientific, Waltham, MA,

173 USA). Quantitative reverse transcription PCR (qRT- PCR) was performed with SsoFast

174 EvaGreen Supermix on a Bio-Rad CFX96 Real-Time System (Bio-Rad, Hercules, CA, USA).

175 The primers used in this study are listed in Supplemental Table 1.

176

177 ROS assays

178 ROS assays were performed as previously described (Pruitt et al. 2015; Wei et al. 2016). Fully-

179 expanded leaves from the same plants used for gene expression assays were cut into $2 \mathrm{~mm}^{2}$

180 pieces and floated in water overnight. Four leaf pieces were pooled and treated with water, 500

$181 \mathrm{nM}$ RaxX21-Y, or $500 \mathrm{nM}$ RaxX21-sY. Each treatment was replicated three times per

182 experiment. Chemiluminescence was recorded in a high-sensitivity TriStar plate reader

183 (Berthold, Germany).

184 


\section{Yeast two-hybrid assays}

186 Yeast two-hybrid assays were performed using the Matchmaker LexA two-hybrid system

187 (Clontech) following the procedures described previously (Chen et al. 2014). The pLexA and

$188 \mathrm{pB} 42 \mathrm{AD}$ vectors were co-transformed into yeast $\mathrm{pEGY48/p8op-lacZ} \mathrm{(Clontech)} \mathrm{using} \mathrm{the}$

189 Frozen-EZ yeast transformation II kit (Zymo Research). To confirm the expression of

190 LexA- and HA-fusion proteins, yeast co-transformed with the appropriate vectors were grown in

191 glucose free SD liquid media overnight with shaking at $30{ }^{\circ} \mathrm{C}$. Cells were pelleted at 3,000 RPM

192 for $5 \mathrm{~min}$, resuspended in $4 \mathrm{x}$ SDS loading buffer, and incubated for $10 \mathrm{~min}$ at $95^{\circ} \mathrm{C}$ to denature

193 proteins.

194

195 Purification of recombinant proteins

196 GST-XA21JK and its variants were expressed in BL21(DE3) pLysS cells (Novagen). Cultures

197 were induced with $0.3 \mathrm{mM}$ IPTG at $23{ }^{\circ} \mathrm{C}$ for $16 \mathrm{~h}$. After $16 \mathrm{~h}$ incubation, cultures were pelleted 198 and resuspended in Cell Wash Buffer (50 mM MOPS, pH 7.5, $150 \mathrm{mM} \mathrm{NaCl}) .250 \mu \mathrm{g} / \mathrm{ml}$

199 lysozyme was added to the cell suspension and incubated at room temperature with shaking for $20030 \mathrm{~min}$. After $30 \mathrm{~min}$, the cell suspension was cooled on ice for at least $30 \mathrm{~min}$. 20X protease 201 inhibitor (10 $\mu \mathrm{M}$ leupeptin, $0.5 \mathrm{mM}$ AEBSF, $1 \mathrm{mM}$ Benzamidine, $2 \mathrm{mM}$ Caproic acid and 10 $202 \mu \mathrm{M}$ E64) was added to the cell suspension and cells were sonicated for $4.5 \mathrm{~min}$ each on ice. The 203 cellular debris was pelleted for $15 \mathrm{~min}$ at $4{ }^{\circ} \mathrm{C}$. The supernatant was transferred into fresh, ice204 cooled $50 \mathrm{ml}$ tubes. GST-fusion proteins were purified using Glutathione-Agarose gel (Sigma205 Aldrich) following the manufacturer's protocol. After elution from the beads, the protein solution 206 was dialyzed against a 1,000× volume of buffer containing $20 \mathrm{mM} \mathrm{Mops,} \mathrm{pH} \mathrm{7.5,} \mathrm{and} 1 \mathrm{mM}$ 207 DTT. 
208 His-Nus-fusion proteins were expressed in BL21 cells. Extraction and purification were 209 described in previous studies (Liu et al. 2002; Schwessinger et al. 2011). Cultures were induced 210 with $0.5 \mathrm{mM} \mathrm{IPTG}$ at $16{ }^{\circ} \mathrm{C}$ overnight. Cells were pelleted and suspended in lysis buffer $(25 \mathrm{mM}$ 211 Tris, $500 \mathrm{mM} \mathrm{NaCl} \mathrm{pH}$ 8.0, $2.5 \mathrm{mM}$ EDTA, $1 \mathrm{mM}$ PMSF, and $1 \mathrm{mg} / \mathrm{mL}$ lysozyme). The 212 bacterial suspension was placed on a shaker for $1 \mathrm{hr}$ at room temperature. Bacterial suspension 213 was pelleted and resuspended on ice in extraction buffer (lysis buffer, $40 \mathrm{mM}$ imidazole, $1 \mathrm{mM}$ $214 \mathrm{MgCl}_{2}, 1 \mathrm{mM}$ DTT, and $\sim 1 \mu \mathrm{L}$ Nuclease). The bacterial suspension was sonicated for $4 \mathrm{~min}$ 215 followed by 10 min shaking on ice. Samples were pelleted and the protein was enriched using a $2160.5 \mu \mathrm{M}$ filter. The fusion proteins were purified using HISTRAP TMFF (GE) according to the 217 manufacturer's protocols. After elution, the concentration of fusion proteins was equalized using $21810 \%$ glycerol solution and stored at $-20{ }^{\circ} \mathrm{C}$ until usage.

\section{In vitro kinase assays}

221 Kinase autophosphorylation assays were performed as described previously (Liu et al. 2002). In 222 brief, purified fusion proteins were incubated in kinase buffer $(50 \mathrm{mM}$ Tris $\cdot \mathrm{HCl}, \mathrm{pH} 7.5,2 \mathrm{mM}$

$223 \mathrm{MgCl}_{2}, 2 \mathrm{mM} \mathrm{MnCl} 2$, and $1 \mathrm{mM}$ DTT), $3 \mu \mathrm{M}$ cold ATP, and $5 \mu \mathrm{Ci}\left[\gamma^{-32} \mathrm{P}\right]-\mathrm{ATP}$ at $30{ }^{\circ} \mathrm{C}$ for 30

224 min with gentle shaking. The reactions were stopped by adding $4 \times$ SDS loading buffer and

225 incubating at $80{ }^{\circ} \mathrm{C}$ for $10 \mathrm{~min}$. Proteins were separated by $10 \%$ SDS-PAGE and the

226 phosphorylation of fusion proteins was analyzed by autoradiography. Densitometry

227 measurements were carried out using imageJ 1.8.0 (Schneider et al. 2012). 


\section{Immunoblotting}

230 Protein extracts from bacteria, yeast, and rice plants were subjected to separation by SDS-PAGE,

231 transferred to polyvinyl difluoride membranes, and immunoblot analysis was performed as

232 described previously (Chen et al. 2014). Epitope-tagged proteins were incubated with the

233 appropriate primary antibodies: anti-GST (Genscript), anti-phosphotyrosine (Genscript), anti-

234 GFP (Santa Cruz Biotech), anti-LexA (Clontech), or anti-HA (Covance). Detection of anti-GST

235 and anti-phosphotyrosine primary antibodies was performed using an Alexa Fluor 680-

236 conjugated secondary antibody (Rockland Immunochemicals, Gilbertsville, PA, USA), and

237 imaged using an Odyssey ${ }^{\circledR}$ Infrared (LI-COR Biosciences) imaging system. All other primary

238 antibodies were incubated with horseradish peroxidase-conjugated anti-mouse (Santa Cruz

239 Biotech), or horseradish peroxidase-conjugated anti-rabbit (GE Healthcare) secondary

240 antibodies. Chemiluminescence substrate (Thermo) was used to detect the horseradish

241 peroxidase-conjugated secondary antibodies by exposure using film or Bio-Rad ChemiDoc ${ }^{\mathrm{TM}}$

242 imaging system. Protein quantification was done using Coomassie Brilliant Blue (CBB) staining.

243 Densitometry measurements were carried out using imageJ 1.8.0 (Schneider et al. 2012).

\section{Results}

246 Identification of XA21 residues required for in vitro tyrosine autophosphorylation.

247 We previously demonstrated that the E. coli-expressed juxtamembrane and kinase 248 domain of XA21 (referred to here as XA21JK, represented in Fig. 1A, Supp. Fig. 1), fused with

249 MBP or GST, autophosphorylates on multiple serine and threonine residues (Liu et al. 2002).

250 More recent studies have demonstrated that other plant LRR-RLKs are also capable of

251 autophosphorylation on tyrosine residues (Macho et al. 2014; Oh et al. 2009). This was observed 
252 by assaying phosphorylation by immunoblotting using anti-phosphotyrosine specific antibodies,

253 which detect tyrosine phosphorylated proteins, but does not allow for the identification of

254 specific Tyr-phosphorylated residues. We applied this technique to XA21JK, to determine if it

255 has tyrosine phosphorylation activity that was previously undetected (Liu et al. 2002), and we

256 observed tyrosine autophosphorylation on XA21JK but not the catalytically inactive variant

257 XA21JK ${ }^{\mathrm{K} 736 \mathrm{E}}$ (Fig. 1B). This result demonstrates that XA21 has tyrosine autophosphorylation

258 activity. A total of nine tyrosine residues, $\operatorname{Tyr}^{698}, \mathrm{Tyr}^{723}, \mathrm{Tyr}^{786}, \mathrm{Tyr}^{829}, \mathrm{Tyr}^{889}, \mathrm{Tyr}^{894}, \mathrm{Tyr}^{907}$,

$259 \mathrm{Tyr}^{909}$, Tyr ${ }^{936}$, are located in the XA21 JK domains (Fig. 1A, Supp. Fig. 1). To determine which

260 of the nine tyrosine residues were potential sites of autophosphorylation, each tyrosine residue

261 was substituted with phenylalanine, which lacks the hydroxyl group required for phosphate

262 transfer. Phosphorylation of the tyrosine variants was monitored by immunoblotting. We found

263 that XA21JK ${ }^{\mathrm{Y} 698 \mathrm{~F}}, \mathrm{XA} 21 \mathrm{JK}^{\mathrm{Y} 786 \mathrm{~F}}, \mathrm{XA} 21 \mathrm{JK}^{\mathrm{Y} 907 \mathrm{~F}}$, and XA21 JK ${ }^{\mathrm{Y} 909 \mathrm{~F}}$ had reduced tyrosine

264 autophosphorylation signals compared with wildtype, suggesting that $\mathrm{Tyr}^{698}{ }^{6} \mathrm{Tyr}^{786}$, Tyr ${ }^{907}$, and

$265 \mathrm{Tyr}^{909}$ are potential sites of tyrosine autophosphorylation in wildtype XA21JK. Tyrosine

266 phosphorylation also appeared slightly reduced in XA21JK ${ }^{\mathrm{Y} 889 \mathrm{~F}}$ and $\mathrm{XA} 21 \mathrm{JK}^{\mathrm{Y} 894 \mathrm{~F}}$, whereas the

267 other tyrosine directed mutants maintained a signal similar to wildtype XA21JK (Fig. 1B).

268 Because the reduced signal associated with $\mathrm{Tyr}^{698}, \mathrm{Tyr}^{786}, \mathrm{Tyr}^{907}$, and $\mathrm{Tyr}^{909}$ were most clearly

269 demonstrable, these four residues were selected for further study.

270

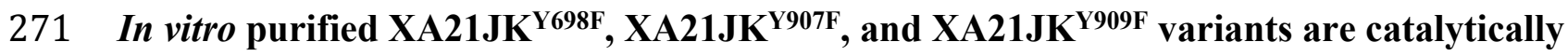

272 active.

273 To determine if $\mathrm{Tyr}^{698}, \mathrm{Tyr}^{786}, \mathrm{Tyr}^{907}$, or $\mathrm{Tyr}^{909}$ are required for XA21 catalytic activity,

274 we expressed the XA21JK, XA21JK ${ }^{\mathrm{D} 82 \mathrm{~N}}, \mathrm{XA} 21 \mathrm{JK}^{\mathrm{YF}}$, and XA21JK ${ }^{\mathrm{YD}}$ variants in E. coli. We 
275 then purified the proteins and incubated each protein in the presence of radiolabeled ATP $([\gamma-$

$\left.\left.276{ }^{32} \mathrm{P}\right]-\mathrm{ATP}\right)$. Autophosphorylation of XA21JK and the mutant variants was determined by the

277 ability of each protein to incorporate radiolabeled phosphate. These experiments indicated that

278 XA21JK possesses kinase activity, whereas XA21JK ${ }^{\mathrm{D} 842 \mathrm{~N}}$ does not (Fig. 2), confirming a

279 previous report (Chen et al. 2014). Autophosphorylation was also observed in $\mathrm{XA} 21 \mathrm{JK}^{\mathrm{Y} 698 \mathrm{~F}}$,

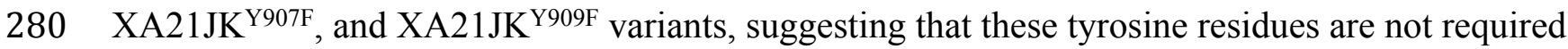

281 for in vitro XA21 catalytic activity (Fig. 2). Furthermore, XA21JK ${ }^{\text {Y907F }}$ was slightly more active

282 and XA21JK ${ }^{\mathrm{Y} 698 \mathrm{~F}}$ was hyperactive compared with XA21JK (Fig. 2). In contrast,

283 autophosphorylation was not detected in XA21 $\mathrm{JK}^{\mathrm{Y} 786 \mathrm{~F}}$, and all four XA21JK ${ }^{\mathrm{YD}}$ variants,

284 suggesting that aspartic acid substitutions at these residues disrupt in vitro XA21 kinase activity

285 (Fig. 2), and that the hydroxyl group of $\operatorname{Tyr}^{786}$ is essential for in vitro kinase activity (Fig. 2).

286

Serine and threonine residues within the juxtamembrane domain of XA21 regulate

287 protein stability (Xu et al. 2006), kinase activity (Chen et al. 2010a), and protein-protein

288 interactions (Chen et al. 2010a; Park et al. 2008). We previously demonstrated that

289 autophosphorylation is reduced in XA21 JK ${ }^{\mathrm{S} 697 \mathrm{~A}}$ and $\mathrm{XA} 21 \mathrm{JK}^{\mathrm{S} 697 \mathrm{D}}$ variants and enhanced in

$290 \mathrm{XA}^{21 J_{K}}{ }^{\mathrm{T} 680 \mathrm{~A}}$ and XA21JK ${ }^{\mathrm{S} 699 \mathrm{~A}}$ proteins (Chen et al. 2010a). We observed similar levels of

291 autophosphorylation in XA21 JK ${ }^{\mathrm{S} 686 \mathrm{~A} / \mathrm{T} 688 \mathrm{~A} / \mathrm{S} 689 \mathrm{~A}}$ and XA21JK. In contrast, XA21JK ${ }^{\mathrm{T} 705 \mathrm{~A}}$ and

292 XA21JK ${ }^{\mathrm{T} 705 \mathrm{E}}$ lost autophosphorylation activity (Chen et al. 2010a). The contribution of these

293 residues in tyrosine autophosphorylation was not tested.

294 To determine whether these previously characterized XA21 variants alter XA21 tyrosine

295 autophosphorylation patterns, we carried out immunoblotting of in vitro recombinant proteins

296 purified from E. coli with Anti-pY antibodies. For these experiments, we tested XA21JK variants

297 that had $\mathrm{Ser}^{697}, \mathrm{Thr}^{705}$, $\mathrm{Thr}^{680}$, or $\mathrm{Ser}^{699}$, replaced with alanine, and XA21JK variants that had 
298 Ser ${ }^{697}$ and $\mathrm{Thr}^{705}$ substituted with aspartate or glutamate (Chen et al. 2010a). We found that the

299 XA21JK ${ }^{\text {S697D }}$ variant displayed tyrosine autophosphorylation similar to wildtype, whereas

$300 \mathrm{XA21JK}^{\mathrm{S} 697 \mathrm{~A}}, \mathrm{XA21 \textrm {JK } ^ { \mathrm { T } 7 0 5 \mathrm { A } } , \mathrm { XA } 2 1 \mathrm { JK } ^ { \mathrm { T } 7 0 5 \mathrm { E } } \text { , and XA21JK }}{ }^{\mathrm{S} 699 \mathrm{~A}}$ variants each displayed reduced

301 tyrosine autophosphorylation (Supp. Fig. 2). Because tyrosine phosphorylation was reduced in

$302 \mathrm{XA} 21 \mathrm{JK}^{\mathrm{S} 697 \mathrm{~A}}$ but not XA21JK ${ }^{\mathrm{S} 697 \mathrm{D}}$, phosphorylation of $\mathrm{Ser}^{697}$ may facilitate autophosphorylation

303 of one or more tyrosine residues in vitro. We were unsuccessful in expressing XA21JK ${ }^{\mathrm{T} 680 \mathrm{~A}}$, so

304 the contribution of $\operatorname{Thr}^{680}$ towards tyrosine phosphorylation was not determined (Supp. Fig. 2).

305

306

307

Transgenic rice plants expressing XA21 ${ }^{\mathrm{YD}}$-GFP variants are susceptible to Xoo.

To determine if $\mathrm{Tyr}^{698}, \mathrm{Tyr}^{786}, \mathrm{Tyr}^{907}$, and $\mathrm{Tyr}^{909}$ affect the immune function of XA21, we

generated Kitaake rice plants expressing a C-terminal GFP-tagged XA21 construct (XA21-GFP)

and XA21-GFP constructs carrying a YD or YF substitution at one of these residues. All

310

constructs were driven by the maize ubiquitin promoter. The GFP tag was previously reported to

not affect XA21 function (Park et al. 2013). At least ten independent rice plants were generated

for XA21-GFP and for each XA21-GFP variant. Expression of XA21-GFP and the XA21-GFP

313 variants were assessed in the $\mathrm{T}_{0}$ generation by immunoblot analysis using anti-GFP antibodies.

314 The $\mathrm{T}_{0}$ transgenic lines with confirmed XA21 expression were inoculated with Xoo (Supp. Fig.

315 3). XA21 $2 \mathrm{YD}_{-} \mathrm{GFP}$ variants were as susceptible to $X o o$ as the Kitaake control plants, while

$316 \mathrm{XA} 21^{\mathrm{YF}}$-GFP variants displayed reduced lesion development.

To further characterize the effect of the mutations, at least two independent lines per

318 tyrosine substitution with confirmed expression of the XA21-GFP variants (XA21 ${ }^{\mathrm{YF}}$-GFP lines

698F-1, 698F-2, Y698F-9, 786F-4, 786F-5, 907F-7, 907F-8, 909F-9, and 909F-11 and XA21 YD_

320 GFP lines 698D-5, 698D-6, 786D-1, 786D-2, 907D-11, 907D-12, 909D-11, and 909D-16) were 
321 advanced to the next generation $\left(\mathrm{T}_{1}\right)$. In the $\mathrm{T}_{1}$ generation, XA21 ${ }^{\mathrm{YF}}-\mathrm{GFP}$ variants, XA21 ${ }^{\mathrm{YD}}-\mathrm{GFP}$

322 variants, XA21-GFP (progeny from homozygous line 5B-5-4-3-1), and Kitaake rice plants were

323 inoculated with Xoo. We found that the XA21-GFP control and each of the XA21 ${ }^{\mathrm{YF}}-\mathrm{GFP}$

324 variants were resistant to Xoo, with limited lesion development. In contrast, each of the XA21 $1^{\mathrm{YD}_{-}}$

325 GFP variants and null-segregants developed water-soaked lesions similar to those observed on

326 the control Kitaake plants. One representative line per tyrosine substitution is presented in Fig. 3.

327 Lesion lengths for at least one additional independent line per tyrosine substitution is presented

328 in Supp. Fig. 4. These results indicate that substitution of tyrosine with aspartic acid, a change

329 that may cause structural alterations to XA21 and is predicted to mimic the negative charge of a

330 phosphorylated tyrosine, disrupts XA21-mediated immunity. This result is consistent with the

331 observation that $\mathrm{XA} 21 \mathrm{JK}^{\mathrm{YD}}$ variants are incapable of autophosphorylation in E. coli.

332 To confirm the expression of XA21-GFP and each of the XA21-GFP variants in the $\mathrm{T}_{1}$

333 plants, protein was extracted from two independent PCR-validated transgenic lines per mutant

334 variant and the Kitaake control line. Similar amounts of protein with the predicted molecular

335 weight of $170 \mathrm{kDa}$ corresponding to XA21-GFP were detected in XA21-GFP, XA21 ${ }^{\mathrm{YF}}$-GFP

336 (lines 698F-1, 698F-9, 786F-4, 786F-5, 907F-7, 907F-8, 909F-9, and 909F-11) and XA21 $1^{\mathrm{YD}_{-}}$

337 GFP (lines 698D-5, 698D-6, 786D-1, 786D-2, 907D-11, 907D-12, 909D-11, and 909D-16)

338 plants. We did not detect a similar sized band in the control Kitaake rice plants that lack XA21-

339 GFP (Fig. 4). These results suggest that although XA21 ${ }^{\mathrm{YD}}$-GFP variants may have structural

340 changes to their protein structure compared to wildtype, the susceptibility of XA21 ${ }^{\mathrm{YD}}$-GFP plants

341 to Xoo was not caused by XA21 protein instability.

342

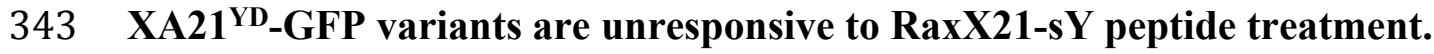


345 responses in the absence of Xoo. Reactive oxygen species (ROS) production and activation of the

346 XA21 genetic markers $O s 04 g 10010$ and $P R 10 b$ are two hallmarks of perception of RaxX21-sY

347 by XA21 (Pruitt et al. 2015; Thomas et al. 2016). Sulfation of RaxX is necessary for recognition, 348 and the non-sulfated RaxX21-Y peptide does not initiate the ROS burst or XA21 marker gene 349 activation (Pruitt et al. 2015). Because XA21 ${ }^{\text {YD }}$-GFP plants are susceptible to Xoo, we 350 hypothesized that tissue from these plants would not respond to RaxX21-sY peptide treatment.

351 To test this hypothesis, we treated leaf clippings from Kitaake, XA21-GFP, and XA21 ${ }^{\text {YD_GFP }}$ 352 variants (lines Y698D-5-14, Y786D-1-2, Y907D-4-2, and Y909D-11-4) with RaxX21-sY, 353 RaxX21-Y, or water, and assessed their immune responses. We found that XA21-GFP leaf 354 clippings displayed a ROS burst (Fig. 5A) and increased expression of Os04g10010 and PR $10 b$ 355 (Fig. 5B) following treatment with RaxX21-sY, but not RaxX21-Y or water. Notably, neither

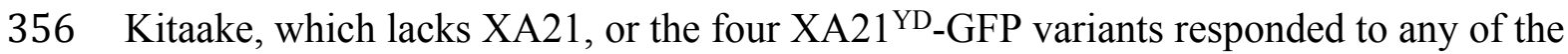
357 treatments (Fig. 5). These results indicate that the XA21 ${ }^{\mathrm{YD}}-\mathrm{GFP}$ variants fail to activate XA21358 mediated defense responses in response to RaxX21-sY, consistent with the observed 359 susceptibility of the XA21 $1^{\mathrm{YD}}$-GFP variants to Xoo.

We next hypothesized that XA2 $11^{\mathrm{YF}}$-GFP variants, which are resistant to $X o o$, would react 361 to RaxX21-sY. To test this hypothesis, leaf clippings of XA21-GFP, Kitaake, and XA21 YF-GFP 362 variants (lines Y698F-2-2, Y786F-5-8, Y907F-7-2, and Y909F-6-5) were treated with RaxX21363 sY, RaxX21-Y, or water. Immune responses were assessed as described for the XA21 ${ }^{\mathrm{YD}_{-} \text {GFP }}$ 364 variants above. We observed that rice plants expressing XA21-GFP and each of the four $365 \mathrm{XA} 21^{\mathrm{YF}}$-GFP variants were responsive to RaxX21-sY treatment, but not RaxX21-Y or water 366 (Fig. 6). This observation supports the conclusion that XA21 ${ }^{\mathrm{YF}}$-GFP variants are able to perceive 
367 RaxX21-sY and initiate an XA21-mediated immune response. Notably, Y698F-2-2 displayed a

368 four-fold higher RaxX-induced ROS (Fig. 6), which may be associated with the hyperactive

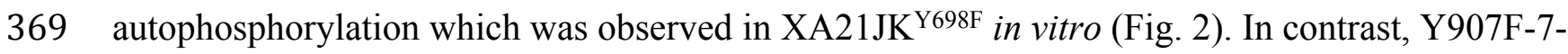

3702 , displayed reduced ROS compared with wildtype (Fig. 6), while XA21JK ${ }^{\mathrm{Y} 907 \mathrm{~F}}$ had only a slight

371 increase in autophosphorylation in vitro (Fig. 2). Similar to Y907F-7-2, Y909F-6-5 also showed

372 reduced ROS compared to wildtype (Fig. 6). Collectively, these results suggest that each tyrosine

373 residue has a different impact on signaling in vivo.

374

375 XA21JK $^{\mathrm{YF}}$ proteins maintain interaction with XB3, XB15, and OsSERK2 in yeast.

376 To determine if XA21 catalytic activity or tyrosine residues regulate protein-protein

377 interactions, we tested the interaction of LexA-XA21JK, LexA-XA21JK ${ }^{\mathrm{D} 842 \mathrm{~N}}$, and the LexA-

$378 \mathrm{XA} 1 \mathrm{JK}^{\mathrm{YF}}$ and LexA-XA21JK ${ }^{\mathrm{YD}}$ variants with HA-XB3, an E3-ubiquitin ligase, HA-XB15, a

379 protein phosphatase, and the co-receptor HA-OsSERK2JMK in yeast (Fig. 7). As previously

380 demonstrated, LexA-XA21JK interacts with HA-XB3, HA-XB15, and HA-OsSERK2JMK

381 (Chen et al. 2014; Park et al. 2008; Wang et al. 2006). LexA-XA21JK ${ }^{\mathrm{D} 842 \mathrm{~N}}$ also maintains

382 interaction with HA-XB3, HA-XB15, and HA-OsSERK2JMK, while no interaction was detected

383 between any of the recombinant proteins and the empty vector controls, LexA-GUS or pB42AD

384 (Fig. 7). This result indicates that XA21 catalytic activity is not required for interaction with

385 XB3, XB15, or OsSERK2.

386 LexA-XA21 $\mathrm{JK}^{\mathrm{YF}}$ variants also maintain interaction with HA-XB3, HA-XB15, and HA-

387 OsSERK2JMK (Fig. 7), indicating that these four tyrosine residues are not required for their

388 interactions. However, the interaction with HA-OsSERK2JMK was disrupted in all four LexA-

$389 \mathrm{XA} 21 \mathrm{JK}^{\mathrm{YD}}$ variants, suggesting that aspartic acid caused a structural change compared with 
390 LexA-XA21JK, or the negative charge of aspartic acid at these four sites disrupt the interaction

391 with HA-OsSERK2JMK in yeast. The interaction with HA-XB3 and HA-XB15 was also

392 disrupted in LexA-XA21JK ${ }^{\mathrm{Y} 698 \mathrm{D}}$ and LexA-XA21JK ${ }^{\mathrm{Y} 909 \mathrm{D}}$, but not LexA-XA21JK ${ }^{\mathrm{Y} 786 \mathrm{D}}$ and

393 LexA-XA21JK ${ }^{\text {Y907D }}$ (Fig. 7).

394

395 Discussion

396 The role of tyrosine phosphorylation in LRR-RLK-mediated responses.

397 Plant LRR-RLKs regulate diverse signaling pathways and the kinase domain is typically

398 at least partially critical for functionality. While many LRR-RLKs were first identified as

399 serine/threonine protein kinases, recent examples have emerged of plant LRR-RLKs with dual

400 specificity; i.e., capable of serine/threonine and tyrosine phosphorylation. For example, tyrosine

401 phosphorylation regulates two Arabidopsis LRR-RLKs, EFR (Macho et al. 2014) and BRI1 (Oh

402 et al. 2009), and the LysM-RLK CERK1 (Liu et al. 2018). In EFR, phosphorylation of Tyr ${ }^{836}$ is

403 required for activation of immune activity (Macho et al. 2014; Oh et al. 2009). A role for the

404 analogous tyrosine residue has also been identified in CERK1 (Liu et al. 2018; Suzuki et al.

405 2018), while BRI1 has a phenylalanine rather than a tyrosine at the corresponding residue

406 (BRI1 ${ }^{\mathrm{F} 996}$, see Supp. Fig. 1). In contrast, phosphorylation of BRI1 ${ }^{\mathrm{Y} 956}$ is thought to inhibit kinase

407 activity (Oh et al. 2012). Tyrosine phosphorylation also regulates BRI1 ${ }^{\text {Y831 }}$, as expression of

$408 \mathrm{BRI1}^{\mathrm{Y} 831 \mathrm{~F}}$ enhances growth, and BRI1 ${ }^{\mathrm{Y} 831 \mathrm{D}}$ recovers wild-type leaf size and flowering time in

409 bril-5 mutant plants (Oh et al. 2009). These results indicate that tyrosine phosphorylation is an

410 important mechanism for regulating plant LRR-RLKs.

411 Previous work with EFR and CERK1 show that individual tyrosine phosphorylation sites

412 which alone do not contribute to overall kinase activity in vitro are indispensable for signaling 
413 (Liu et al. 2018; Macho et al. 2014; Suzuki et al. 2016; Suzuki et al. 2018). In this study, we

414 observed that XA2 $1^{\mathrm{Y} 829 \mathrm{~F}}$ does not reduce the detectable levels of autophosphorylated tyrosine in

415 vitro, as was observed with $\mathrm{EFR}^{\mathrm{Y} 836 \mathrm{~F}}$ (Macho et al 2014), and was not selected for further

416 characterization. Thus, it would be very interesting to perform an in planta mutagenesis analysis

417 of XA2 $1^{\mathrm{Y} 829}$ in order to compare regulatory mechanisms of XA21 and EFR, given that signaling

418 components for XA21- and EFR-mediated immunity are known to be shared (Chen et al. 2014;

419 Holton et al. 2015; Schwessinger et al. 2015; Thomas et al. 2018).

420

While previous studies have focused primarily on the importance of tyrosine

421 phosphorylation by studying the effect of tyrosine phosphorylation absence (using YF

422 mutations), less attention has been given to studying the effect of tyrosine phosphorylation

423 presence (using phosphomimetic YD mutations). This is in part because phosphorylation

424 mimicking mutations do not perfectly mimic phosphorylated amino acids, and may cause steric

425 alterations or structural changes (Corbit et al. 2003; Liu et al. 2018; Paleologou et al. 2008;

426 Suzuki et al. 2018). In this study, we demonstrate that aspartic acid substitution of XA21 tyrosine

427 residues $\mathrm{Tyr}^{698}, \mathrm{Tyr}^{786}, \mathrm{Tyr}^{907}$, and $\mathrm{Tyr}^{909}$, disrupt catalytic activity of XA21, interaction with

428 essential signaling proteins, and XA21-mediated immunity. Further studies are needed to assess

429 if autophosphorylation of specific XA21 tyrosine residues occurs in vivo.

430

431 The mechanism regulating the gatekeeper residue differs between kinases.

432 In 1995, Hanks and Hunter identified a "gatekeeper" residue, located in kinase

433 subdomain $\mathrm{V}$, adjacent to the hinge that connects the $\mathrm{N}$-and $\mathrm{C}$-lobes. This residue is located

434 immediately distal to the active site and regulates access to the ATP binding pocket (Hanks \&

435 Hunter 1995; Paul et al. 2014). In plant kinases, tyrosine residues are highly conserved at the 
436 gatekeeper position. For example, a previous study found that $83 \%$ of aligned Arabidopsis

437 RLK/RLCK family proteins contained tyrosine at the gatekeeper position (Klaus-Heisen et al.

438 2011). The gatekeeper tyrosine residue is present in EFR, BRI1, and XA21 (EFR ${ }^{\mathrm{Y} 791}$, BRI1 ${ }^{\mathrm{Y} 956 \text {, }}$ 439 and $\mathrm{XA} 21 \mathrm{JK}^{\mathrm{Y} 786}$ ) (Supp. Fig. 1). In contrast, another Arabidopsis LRR-RLK, FLS2, contains a

440 leucine residue at the gatekeeper position, suggesting that the gatekeeper tyrosine residue is not a 441 requirement for biological activity in plant LRR-RLKs.

442 Studies assessing the substitution of the gatekeeper tyrosine residue with phenylalanine

443 have found contrasting effects on catalytic activity. For example, a phenylalanine substitution

444 mutant of the conserved gatekeeper tyrosine in the Symbiosis Receptor Kinase from Arachis

445 hypogaea (AhSYMRK $\left.{ }^{\mathrm{Y} 670 \mathrm{~F}}\right)$ is catalytically active, suggesting it is not involved in

446 phosphotransfer (Samaddar et al. 2013). In contrast, the lysin motif domain-containing receptor-

447 like kinase-3 (LYK3 $\left.{ }^{\mathrm{Y} 390 \mathrm{~F}}\right)$ in Medicago truncatula only maintains partial kinase activity (Klaus-

448 Heisen et al. 2011), and autophosphorylation activity is completely abolished in BRI1 ${ }^{\mathrm{Y} 956 \mathrm{~F}}$ (Oh et

449 al. 2009). In this study, we identified XA2 $21^{\mathrm{Y} 786}$, the gatekeeper tyrosine residue, as one of three

450 tyrosine residues in the XA21 kinase domain predicted to function in tyrosine phosphorylation.

451 Like BRI1 ${ }^{\mathrm{Y} 956 \mathrm{~F}}$, we found that XA21 JK ${ }^{\mathrm{Y} 786 \mathrm{~F}}$ autophosphorylation is significantly reduced (Fig.

452 2), suggesting the gatekeeper tyrosine residue is important for catalytic activity in XA21.

453 Phosphorylation of the gatekeeper residues also has varying effects on biological activity

454 between kinases. For example, BRI1 ${ }^{\mathrm{Y} 956 \mathrm{~F}}$ is inactive in vivo, whereas $A h \mathrm{SYMRK}^{\mathrm{Y} 670 \mathrm{~F}}$,

$455 M t \mathrm{LYK}^{\mathrm{Y} 390 \mathrm{~F}}$, and $\mathrm{XA}_{2} 1^{\mathrm{Y} 786 \mathrm{~F}_{-} \mathrm{GFP}}$ are all biologically active. $\mathrm{EFR}^{\mathrm{Y} 791 \mathrm{~F}}$ is also able to initiate

456 EFR-mediated immunity. However, catalytic activity of EFR ${ }^{\mathrm{Y} 791 \mathrm{~F}}$ has not been established

457 (Macho et al. 2014). A follow up study in AhSYMRK utilizing the phosphomimetic mutant

$458 A^{A} \mathrm{SYMRK}^{\mathrm{Y} 670 \mathrm{E}}$ suggested that phosphorylation of the gatekeeper residue is autoinhibitory, 
459 rather than essential for activity (Paul et al. 2014). Our study suggests that XA21 functions

460 similarly, as XA2 $1^{\mathrm{Y} 786 \mathrm{~F}}$-GFP is biologically active, while $\mathrm{XA} 21^{\mathrm{Y} 786 \mathrm{D}}$-GFP is not. Collectively,

461 our work provides further evidence that the mechanism regulating the gatekeeper tyrosine

462 residue differs between kinases and must be assessed on a case-by-case basis.

463

464 XA21 catalytic activity is not required for interaction with OsSERK2

465

Interaction between Arabidopsis LRR-RLKs such as FLS2 and EFR and the co-receptor

466

BAK1 are ligand dependent, occurring rapidly upon perception of their cognate bacterial

467 peptides (Schulze et al. 2010). While an active kinase is required for biological activity, it is not

468 required for ligand-induced complex formation with BAK1 (Schwessinger et al. 2011). In

469 contrast, catalytic activity of BRI1 is required for complex formation with BAK1 (Wang et al.

470 2008). In rice, the co-receptor OsSERK2 forms interactions with three different rice LRR-RLKs,

471 XA21, XA3, and OsFLS2 (Chen et al. 2014). Unlike complex formation between BAK1 and the

472 LRR-RLKs of Arabidopsis, XA21 forms a constitutive complex with OsSERK2 in the absence

473 of bacterial elicitors (Chen et al. 2014). The XA21-OsSERK2 complex was suspected to be

474 kinase activity dependent, as catalytically inactive $\mathrm{XA} 21 \mathrm{JK}^{\mathrm{K} 736 \mathrm{E}}$ fails to interact with

475 OsSERK2JMK in yeast (Chen et al. 2014). However, in this study we identified an additional

476 XA21 variant, XA21 JK ${ }^{\mathrm{D} 842 \mathrm{~N}}$, which is catalytically inactive, yet maintains interaction with

477 OsSERK2JMK in yeast, indicating that catalytic activity is not the sole determinant of XA21-

478 OsSERK2 interaction. Alternatively, it is possible that the replacement of the positive charged

479 lysine with the negative charge of glutamate in $\mathrm{XA} 21 \mathrm{JK}^{\mathrm{K} 736 \mathrm{E}}$ may interfere with the interaction

480 between proteins, or be causing a structural change in the protein. Thus, we suggest that future

481 claims regarding the necessity of catalytic activity on protein-protein interactions should ideally 
482 include the use of multiple catalytically inactive variants, as the impacts of individual variants 483 can differ.

484

While it has been demonstrated that the mechanism controlling LRR-RLK and co-

485 receptor interaction differs between rice and Arabidopsis, the mechanism controlling signaling

486 initiation is currently unknown. Biological activity of FLS2 and EFR both require active kinases.

487 Similarly, rice expressing XA2 $1^{\mathrm{K} 736 \mathrm{E}}$ are only partially resistant to Xoo (Andaya \& Ronald

488 2003). However, XA21 may differ from FLS2 and EFR, as rice expressing XA21 Y786F_GFP are

489 resistant to Xoo (Fig. 3), suggesting that the ability of XA21 to autophosphorylate in vitro does

490 not correlate with XA21-mediated resistance in planta. We hypothesize that OsSERK2

491 transphosphorylation of XA2 $1^{\mathrm{Y} 786 \mathrm{~F}}$-GFP is sufficient to confer resistance to Xoo, whereas the

492 interaction between OsSERK2 and XA21JK ${ }^{\mathrm{K} 736 \mathrm{E}}$ is weaker (Chen et al. 2014), and thus

$493 \mathrm{XA} 21 \mathrm{JK}^{\mathrm{K} 736 \mathrm{E}}$ is only sufficient to provide the partial resistance observed previously (Andaya \&

494 Ronald 2003). In agreement with this hypothesis, it was demonstrated that OsSERK2JMK is able

495 to transphosphorylate XA21 JK ${ }^{\mathrm{K} 736 \mathrm{E}}$ despite not being able to observe a direct interaction (Chen

496 et al. 2014). However, we cannot rule out that XA2 $1^{\mathrm{Y} 786 \mathrm{~F}}$-GFP has additional in vivo kinase

497 activity that was not observable in vitro (Fig. 2). Collectively, these results suggest that the

498 OsSERK2-XA21 interaction is required for full resistance to $\mathrm{Xoo}$, but the interaction is not

499 dependent on these four specific tyrosine residues. Furthermore, the mechanism regulating

500 activation of XA21 differs from previously studied LRR-RLKs including BRI1, EFR, or FLS2.

501

502 Conclusions

503 While many plant LRR-RLKs were first identified as serine/threonine protein kinases, recent

504 examples have emerged of plant LRR-RLKs capable of serine/threonine and tyrosine 
505 phosphorylation. In this study, we determined that XA21 is capable of performing in vitro

506 tyrosine autophosphorylation. However, the four tyrosine residues which we analyzed are not

507 required for activation of XA21-mediated immune responses, or interaction with predicted XA21

508 signaling components including the co-receptor OsSERK2.

509

\section{Acknowledgements}

511 We thank Dr. Benjamin Schwessinger for helpful discussions and technical assistance in

512 performing kinase autophosphorylation assays and Dr. Gitta Coaker for her supportive

513 discussions of the manuscript and the application of statistical methods. We express our gratitude

514 to Dr. Christopher Harvey for helping with the assembly of raw data in preparation for peer

515 review.

516 


\section{References}

518

519

520

521

522

523

524

525

526

527

528

529

530

531

532

533

534

535

536

537

538

539

540

541

542

543

544

545

546

547

548

549

550

551

552

553

554

555

556

557

558

559

560

561
Andaya CB, and Ronald PC. 2003. A catalytically impaired mutant of the rice Xa21 receptor kinase conferspartial resistance toXanthomonas oryzae pv oryzae.

Chen X, Chern M, Canlas PE, Jiang C, Ruan D, Cao P, and Ronald PC. 2010a. A conserved threonine residue in the juxtamembrane domain of the XA21 pattern recognition receptor is critical for kinase autophosphorylation and XA21-mediated immunity. $J$ Biol Chem 285:10454-10463. 10.1074/jbc.M109.093427

Chen X, Chern M, Canlas PE, Ruan D, Jiang C, and Ronald PC. 2010b. An ATPase promotes autophosphorylation of the pattern recognition receptor XA21 and inhibits XA21mediated immunity. Proc Natl Acad Sci U S A 107:8029-8034. 10.1073/pnas.0912311107

Chen X, Zuo S, Schwessinger B, Chern M, Canlas PE, Ruan D, Zhou X, Wang J, Daudi A, Petzold CJ, Heazlewood JL, and Ronald PC. 2014. An XA21-associated kinase (OsSERK2) regulates immunity mediated by the XA21 and XA3 immune receptors. Mol Plant 7:874-892. 10.1093/mp/ssu003

Chern M, Canlas PE, Fitzgerald HA, and Ronald PC. 2005. Rice NRR, a negative regulator of disease resistance, interacts with Arabidopsis NPR1 and rice NH1. Plant J 43:623635. 10.1111/j.1365-313X.2005.02485.X

Chinchilla D, Zipfel C, Robatzek S, Kemmerling B, Nurnberger T, Jones JD, Felix G, and Boller T. 2007. A flagellin-induced complex of the receptor FLS2 and BAK1 initiates plant defence. Nature 448:497-500. 10.1038/nature05999

Corbit KC, Trakul N, Eves EM, Diaz B, Marshall M, and Rosner MR. 2003. Activation of Raf-1 signaling by protein kinase $C$ through a mechanism involving Raf kinase inhibitory protein. J Biol Chem 278:13061-13068. 10.1074/jbc.M210015200

Gomez-Gomez L, and Boller T. 2000. FLS2: an LRR receptor-like kinase involved in the perception of the bacterial elicitor flagellin in Arabidopsis. Mol Cell 5:1003-1011.

Hanks SK, and Hunter T. 1995. Protein kinases 6. The eukaryotic protein kinase superfamily: kinase (catalytic) domain structure and classification. FASEB J 9:576596.

Holton N, Nekrasov V, Ronald PC, and Zipfel C. 2015. The phylogenetically-related pattern recognition receptors EFR and XA21 recruit similar immune signaling components in monocots and dicots. PLoS Pathog 11:e1004602. 10.1371/journal.ppat.1004602

Klaus-Heisen D, Nurisso A, Pietraszewska-Bogiel A, Mbengue M, Camut S, Timmers T, Pichereaux C, Rossignol M, Gadella TW, Imberty A, Lefebvre B, and Cullimore JV. 2011. Structure-function similarities between a plant receptor-like kinase and the human interleukin-1 receptor-associated kinase-4. J Biol Chem 286:11202-11210. 10.1074/jbc.M110.186171

Liu GZ, Pi LY, Walker JC, Ronald PC, and Song WY. 2002. Biochemical characterization of the kinase domain of the rice disease resistance receptor-like kinase XA21. J Biol Chem 277:20264-20269. 10.1074/jbc.M110999200

Liu J, Liu B, Chen S, Gong BQ, Chen L, Zhou Q, Xiong F, Wang M, Feng D, Li JF, Wang HB, and Wang J. 2018. A Tyrosine Phosphorylation Cycle Regulates Fungal Activation of a Plant Receptor Ser/Thr Kinase. Cell Host Microbe 23:241-253 e246. 10.1016/j.chom.2017.12.005 
562 Macho AP, Lozano-Duran R, and Zipfel C. 2015. Importance of tyrosine phosphorylation in

563

564

565

566

567

568

569

570

571

572

573

574

575

576

577

578

579

580

581

582

583

584

585

586

587

588

589

590

591

592

593

594

595

596

597

598

599

600

601

602

603

604

605

606

607 receptor kinase complexes. Trends Plant Sci 20:269-272. 10.1016/j.tplants.2015.02.005

Macho AP, Schwessinger B, Ntoukakis V, Brutus A, Segonzac C, Roy S, Kadota Y, Oh MH, Sklenar J, Derbyshire P, Lozano-Duran R, Malinovsky FG, Monaghan J, Menke FL, Huber SC, He SY, and Zipfel C. 2014. A bacterial tyrosine phosphatase inhibits plant pattern recognition receptor activation. Science 343:1509-1512.

10.1126/science.1248849

Nakagami H, Sugiyama N, Mochida K, Daudi A, Yoshida Y, Toyoda T, Tomita M, Ishihama Y, and Shirasu K. 2010. Large-scale comparative phosphoproteomics identifies conserved phosphorylation sites in plants. Plant Physiol 153:1161-1174. 10.1104/pp.110.157347

Nolen B, Taylor S, and Ghosh G. 2004. Regulation of protein kinases; controlling activity through activation segment conformation. Mol Cell 15:661-675. 10.1016/j.molcel.2004.08.024

Oh MH, Clouse SD, and Huber SC. 2012. Tyrosine Phosphorylation of the BRI1 Receptor Kinase Occurs via a Post-Translational Modification and is Activated by the Juxtamembrane Domain. Front Plant Sci 3:175. 10.3389/fpls.2012.00175

Oh MH, Wang X, Kota U, Goshe MB, Clouse SD, and Huber SC. 2009. Tyrosine phosphorylation of the BRI1 receptor kinase emerges as a component of brassinosteroid signaling in Arabidopsis. Proc Natl Acad Sci U S A 106:658-663. 10.1073/pnas.0810249106

Paleologou KE, Schmid AW, Rospigliosi CC, Kim HY, Lamberto GR, Fredenburg RA, Lansbury PT, Jr., Fernandez CO, Eliezer D, Zweckstetter M, and Lashuel HA. 2008. Phosphorylation at Ser-129 but not the phosphomimics S129E/D inhibits the fibrillation of alpha-synuclein. J Biol Chem 283:16895-16905. 10.1074/jbc.M800747200

Park CJ, Lee SW, Chern M, Sharma R, Canlas PE, Song MY, Jeon JS, and Ronald PC. 2010. Ectopic expression of rice Xa21 overcomes developmentally controlled resistance to Xanthomonas oryzae pv. oryzae. Plant Sci 179:466-471. 10.1016/j.plantsci.2010.07.008

Park CJ, Peng Y, Chen X, Dardick C, Ruan D, Bart R, Canlas PE, and Ronald PC. 2008. Rice $\mathrm{XB15}$, a protein phosphatase $2 \mathrm{C}$, negatively regulates cell death and XA21-mediated innate immunity. PLoS Biol 6:e231. 10.1371/journal.pbio.0060231

Park CJ, Sharma R, Lefebvre B, Canlas PE, and Ronald PC. 2013. The endoplasmic reticulumquality control component SDF2 is essential for XA21-mediated immunity in rice. Plant Sci 210:53-60. 10.1016/j.plantsci.2013.05.003

Paul A, Samaddar S, Bhattacharya A, Banerjee A, Das A, Chakrabarti S, and DasGupta M. 2014. Gatekeeper tyrosine phosphorylation is autoinhibitory for Symbiosis Receptor Kinase. FEBS Lett 588:2881-2889. 10.1016/j.febslet.2014.06.056

Pruitt RN, Schwessinger B, Joe A, Thomas N, Liu F, Albert M, Robinson MR, Chan LJ, Luu DD, Chen H, Bahar O, Daudi A, De Vleesschauwer D, Caddell D, Zhang W, Zhao X, Li X, Heazlewood JL, Ruan D, Majumder D, Chern M, Kalbacher H, Midha S, Patil PB, Sonti RV, Petzold CJ, Liu CC, Brodbelt JS, Felix G, and Ronald PC. 2015. The rice immune receptor XA21 recognizes a tyrosine-sulfated protein from a Gram-negative bacterium. Sci Adv 1:e1500245. 10.1126/sciadv.1500245 
608

609

610

611

612

613

614

615

616

617

618

619

620

621

622

623

624

625

626

627

628

629

630

631

632

633

634

635

636

637

638

639

640

641

642

643

644

645

646

647

648

649

650

651

Samaddar S, Dutta A, Sinharoy S, Paul A, Bhattacharya A, Saha S, Chien KY, Goshe MB, and DasGupta M. 2013. Autophosphorylation of gatekeeper tyrosine by symbiosis receptor kinase. FEBS Lett 587:2972-2979. 10.1016/j.febslet.2013.07.050

Schneider CA, Rasband WS, and Eliceiri KW. 2012. NIH Image to ImageJ: 25 years of image analysis. Nat Methods 9:671-675.

Schulze B, Mentzel T, Jehle AK, Mueller K, Beeler S, Boller T, Felix G, and Chinchilla D. 2010. Rapid heteromerization and phosphorylation of ligand-activated plant transmembrane receptors and their associated kinase BAK1.J Biol Chem 285:94449451. 10.1074/jbc.M109.096842

Schwessinger B, Bahar O, Thomas N, Holton N, Nekrasov V, Ruan D, Canlas PE, Daudi A, Petzold CJ, Singan VR, Kuo R, Chovatia M, Daum C, Heazlewood JL, Zipfel C, and Ronald PC. 2015. Transgenic expression of the dicotyledonous pattern recognition receptor EFR in rice leads to ligand-dependent activation of defense responses. PLoS Pathog 11:e1004809. 10.1371/journal.ppat.1004809

Schwessinger B, Roux M, Kadota Y, Ntoukakis V, Sklenar J, Jones A, and Zipfel C. 2011. Phosphorylation-dependent differential regulation of plant growth, cell death, and innate immunity by the regulatory receptor-like kinase BAK1. PLoS Genet 7:e1002046. 10.1371/journal.pgen.1002046

Suzuki M, Shibuya M, Shimada H, Motoyama N, Nakashima M, Takahashi S, Suto K, Yoshida I, Matsui S, Tsujimoto N, Ohnishi M, Ishibashi Y, Fujimoto Z, Desaki Y, Kaku H, Kito K, and Shibuya N. 2016. Autophosphorylation of Specific Threonine and Tyrosine Residues in Arabidopsis CERK1 is Essential for the Activation of Chitin-Induced Immune Signaling. Plant Cell Physiol 57:2312-2322. 10.1093/pcp/pcw150

Suzuki M, Watanabe T, Yoshida I, Kaku H, and Shibuya N. 2018. Autophosphorylation site Y428 is essential for the in vivo activation of CERK1. Plant Signal Behav 13:e1435228. 10.1080/15592324.2018.1435228

Takai R, Isogai A, Takayama S, and Che FS. 2008. Analysis of flagellin perception mediated by flg22 receptor OsFLS2 in rice. Mol Plant Microbe Interact 21:1635-1642. 10.1094/MPMI-21-12-1635

Thomas NC, Oksenberg N, Liu F, Caddell D, Nalyvayko A, Nguyen Y, Schwessinger B, and Ronald PC. 2018. The rice XA21 ectodomain fused to the Arabidopsis EFR cytoplasmic domain confers resistance to Xanthomonas oryzae pv. oryzae. PeerJ 6:e4456. 10.7717/peerj.4456

Thomas NC, Schwessinger B, Liu F, Chen H, Wei T, Nguyen YP, Shaker IWF, and Ronald PC. 2016. XA21-specific induction of stress-related genes following Xanthomonas infection of detached rice leaves. PeerJ 4:e2446.10.7717/peerj.2446

Wang X, Kota U, He K, Blackburn K, Li J, Goshe MB, Huber SC, and Clouse SD. 2008. Sequential transphosphorylation of the BRI1/BAK1 receptor kinase complex impacts early events in brassinosteroid signaling. Dev Cell 15:220-235. 10.1016/j.devcel.2008.06.011

Wang YS, Pi LY, Chen X, Chakrabarty PK, Jiang J, De Leon AL, Liu GZ, Li L, Benny U, Oard J, Ronald PC, and Song WY. 2006. Rice XA21 binding protein 3 is a ubiquitin ligase required for full Xa21-mediated disease resistance. Plant Cell 18:3635-3646. $10.1105 /$ tpc.106.046730 
652 Wei T, Chen TC, Ho YT, and Ronald PC. 2016. Mutation of the rice XA21 predicted nuclear 653 localization sequence does not affect resistance to Xanthomonas oryzae pv. oryzae. $654 \quad$ PeerJ 4:e2507. 10.7717/peerj.2507

655 Xiang Y, Cao Y, Xu C, Li X, and Wang S. 2006. Xa3, conferring resistance for rice bacterial 656 blight and encoding a receptor kinase-like protein, is the same as Xa26. Theor Appl 657 Genet 113:1347-1355. 10.1007/s00122-006-0388-x

658 659 660 661 662 663 664 665

Xu WH, Wang YS, Liu GZ, Chen X, Tinjuangjun P, Pi LY, and Song WY. 2006. The autophosphorylated Ser686, Thr688, and Ser689 residues in the intracellular juxtamembrane domain of XA21 are implicated in stability control of rice receptorlike kinase. Plant J 45:740-751. 10.1111/j.1365-313X.2005.02638.x

Zipfel C, Kunze G, Chinchilla D, Caniard A, Jones JD, Boller T, and Felix G. 2006. Perception of the bacterial PAMP EF-Tu by the receptor EFR restricts Agrobacterium-mediated transformation. Cell 125:749-760. 10.1016/j.cell.2006.03.037 


\section{Figure 1}

XA21 is autophosphorylated on tyrosine residues in vitro.

(A) Representation of the XA21 juxtamembrane (JM), kinase subdomains (I-XI), and Cterminal region (CT) (XA21JK). Full-length XA21 has N-terminal leucine-rich repeats and a transmembrane domain that are not depicted. The positions of the nine XA21JK tyrosine residues $(Y)$ are indicated in black. The conserved lysine $(K)$ required for kinase activity and aspartate (D) required for phospho-transfer are labeled in orange. Previously studied XA21 JM Threonine (T) and Serine (S) residues are depicted in blue. (B) Immunoblots of wildtype (WT) GST-tagged XA21 (GST-XA21JK) and GST-XA21JK with lysine modified to glutamine (GSTXA21JK ${ }^{\mathrm{K} 736 \mathrm{E}}$ ) and WT GST-XA21JK and variants with individual tyrosine residues modified to phenylalanine (Y698F, Y723F, Y786F, Y829F, Y889F, Y894F, Y907F, Y909F, Y936F). Tyrosinespecific phosphorylation was detected by immunoblotting with anti-phosphotyrosine antibodies (anti-pY). Equal loading of proteins was confirmed by immunoblotting with an antiGST antibody and/or Coomassie Brilliant Blue (CBB) staining of the membrane. Densitometry measurements shown below the panel represent the ratio between phosphotyrosine and GST signals normalized to WT GST-XA21JK. The above experiments were performed twice with similar results. 


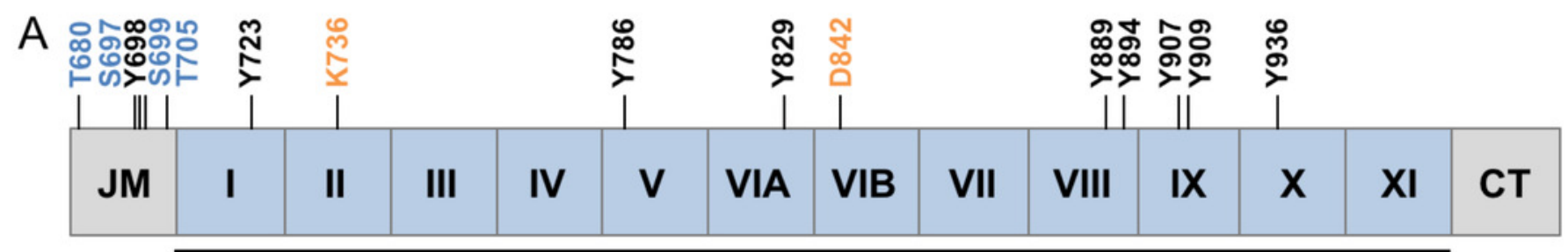

Kinase Domain

B

\section{GST-XA21JK}

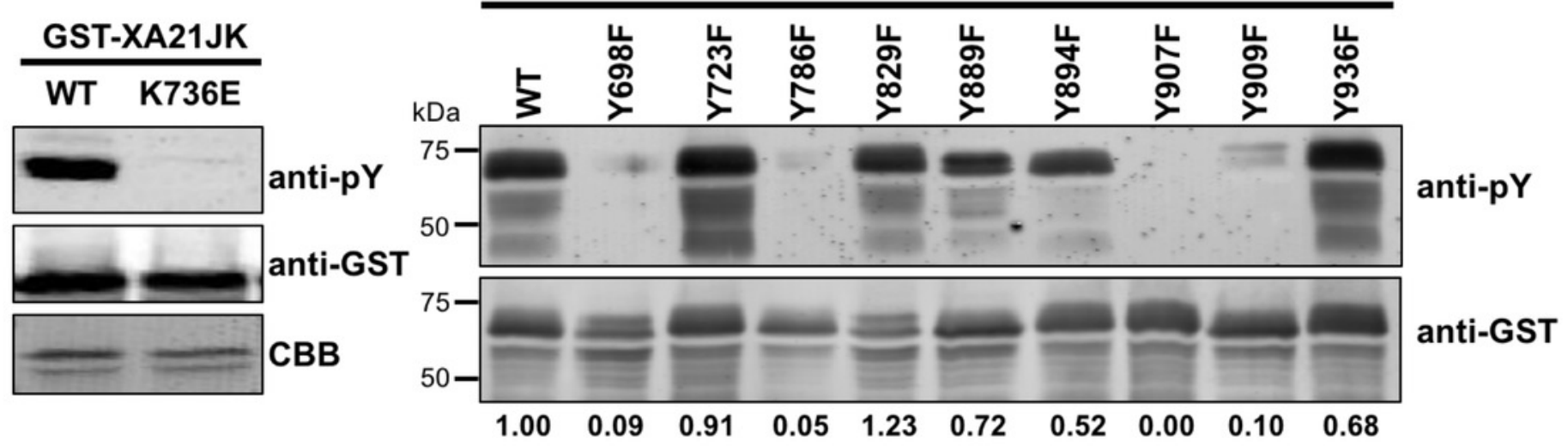




\section{Figure 2}

Kinase autophosphorylation activity is lost in certain His-Nus-XA21JK variants.

Kinase autophosphorylation assay of purified E. coli expressed His-Nus-XA21JK wild-type (WT) and variant proteins (Y698F, Y698D, Y786F, Y786D, D842N, Y907F, Y907D, Y909F, Y909D). Kinase autophosphorylation was assessed by $[\mathrm{\gamma}-32 \mathrm{P}]-\mathrm{ATP}\left({ }^{32} \mathrm{P}\right)$ incorporation (see materials and methods). The autoradiogram (top) and Coomassie Brilliant Blue (CBB) stained gel (bottom) of the same gel are shown. His-Nus-XA21JK ${ }^{\mathrm{D} 842 \mathrm{~N}}$ is catalytically inactive and is included as a negative control. Densitometry measurements shown below the panel represent the ratio between ${ }^{32} \mathrm{P}$ signal and CBB stain normalized to WT GST-XA21JK. This experiment was performed twice using independent protein purifications with similar results.

\section{His-Nus-XA21JK}

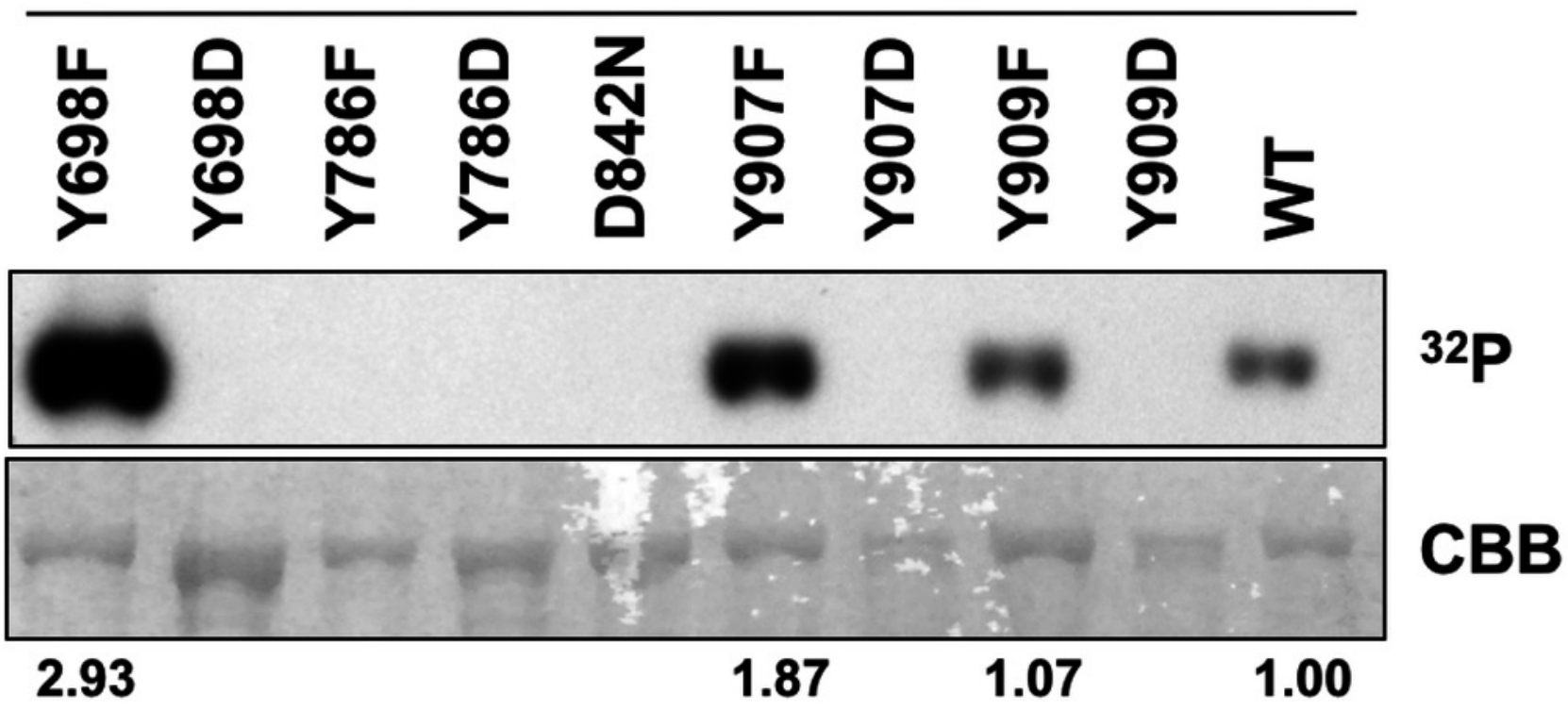




\section{Figure 3}

Rice plants expressing XA21 ${ }^{\mathrm{YD}}$-GFP variants are susceptible to $\mathrm{XoO}$.

Lesion length of Kitaake, XA21-GFP, and $\mathrm{T}_{1}$ generation mutant plants 14 days after

inoculation with $X_{\circ o}$. Bars indicate the mean lesion length and standard deviation of a single plant with four to eleven leaves inoculated with $X O O O D=0.5$. Different letters indicate a significant difference in lesion length $(P<0.05$, Kruskal-Wallis test, Dunn's post-hoc test with Benjamini-Hochberg correction). Gray bars indicate the presence of the XA21-GFP construct as assessed by PCR (Primers in Supplemental Table 1). White bars indicate null-segregants. Shown is one representative transgenic line per tyrosine substitution. At least one additional independent transgenic line per tyrosine substitution was inoculated with similar results (Supp. Fig. 4). This experiment was performed 3 times with similar results. Two representative leaves per transgenic line were photographed at the time of lesion measurement (right panels). The top and bottom panels were inoculated on separate dates. 

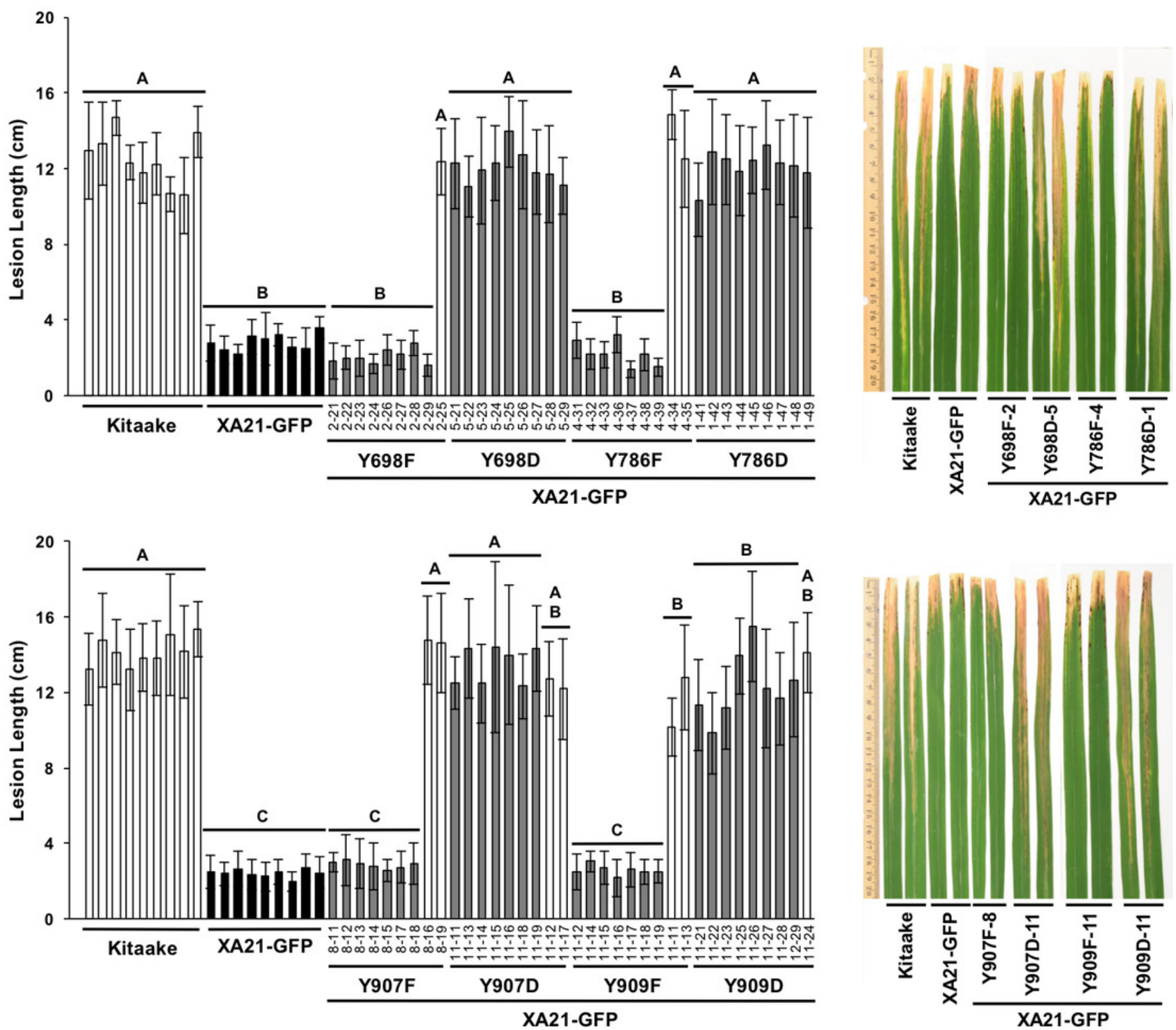
Figure 4

XA21-GFP proteins are expressed in rice leaves.

Immunoblotting of XA21-GFP and mutant variants was performed using anti-GFP antibodies (top panel). A signal corresponding to the expected molecular weight of XA21-GFP was detected in XA21-GFP and two independently-transformed lines per tyrosine substitution. No signal was detected in Kitaake, which lacks Xa21. Equal loading of proteins was confirmed by Coomassie Brilliant Blue (CBB) staining of the membrane after immunoblotting (lower panel). This experiment was repeated three times with similar results.
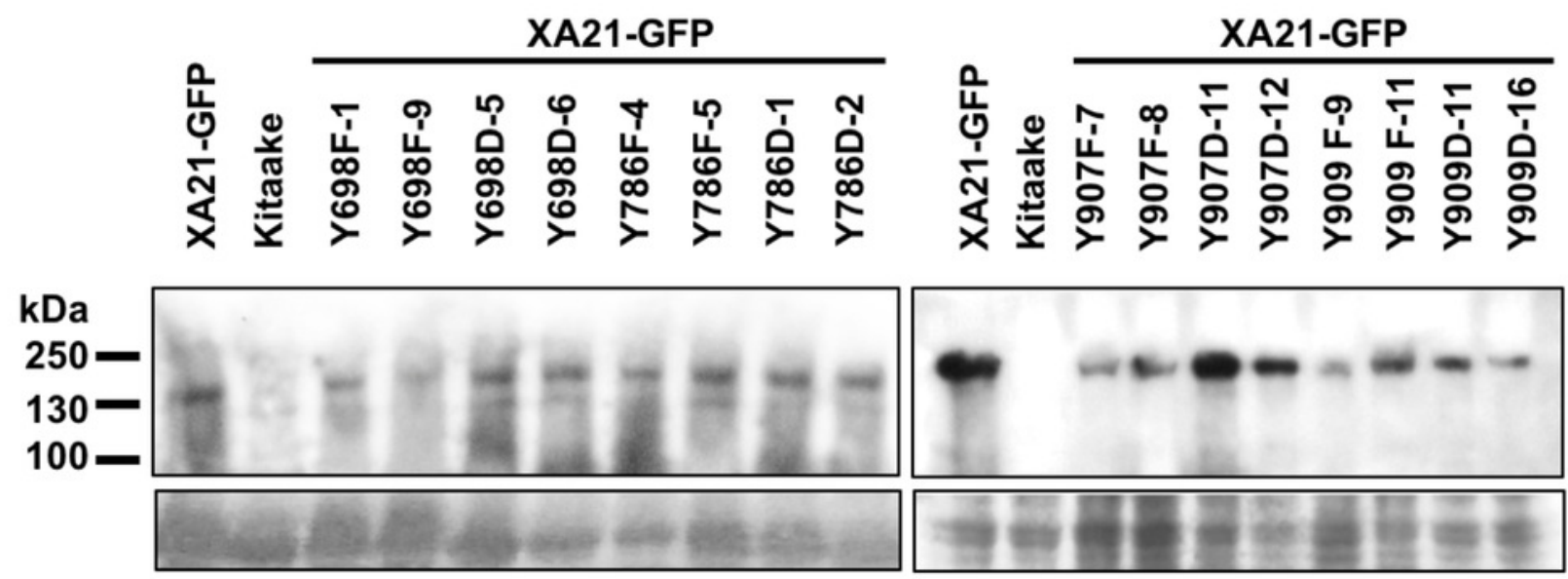

anti-GFP

CBB 


\section{Figure 5}

XA21 YD-GFP variants do not respond to sulfated RaxX.

(A) ROS production in rice leaves of Kitaake, XA21-GFP, and XA21 ${ }^{\text {YD }}$-GFP variants six hours after treatment with water (mock, blue), $1 \mu \mathrm{M}$ RaxX21-Y (yellow) or $1 \mu \mathrm{M}$ RaxX21-sY (gray). Data points depict the mean \pm standard error $(n=3)$. Each panel depicts a different plant background from a single experiment. RLU, relative light units. (B) Gene expression of Os04g10010 (left) and PR10b (Os12g36850, right) in rice leaves of Kitaake, XA21-GFP, and XA21 ${ }^{Y D}$-GFP variants six hours after treatment with water (mock), $500 \mathrm{nM}$ RaxX21-Y, or 500 nM RaxX21-sY. Bars depict the means \pm standard deviation $(n=3)$ of expression level normalized to mock-treated XA21-GFP. "** indicates a significant difference in gene expression compared to the mock treatment $(P<0.05$, ANOVA, Tukey-HSD). Similar results were obtained from one additional independent transgenic line per tyrosine variant. 

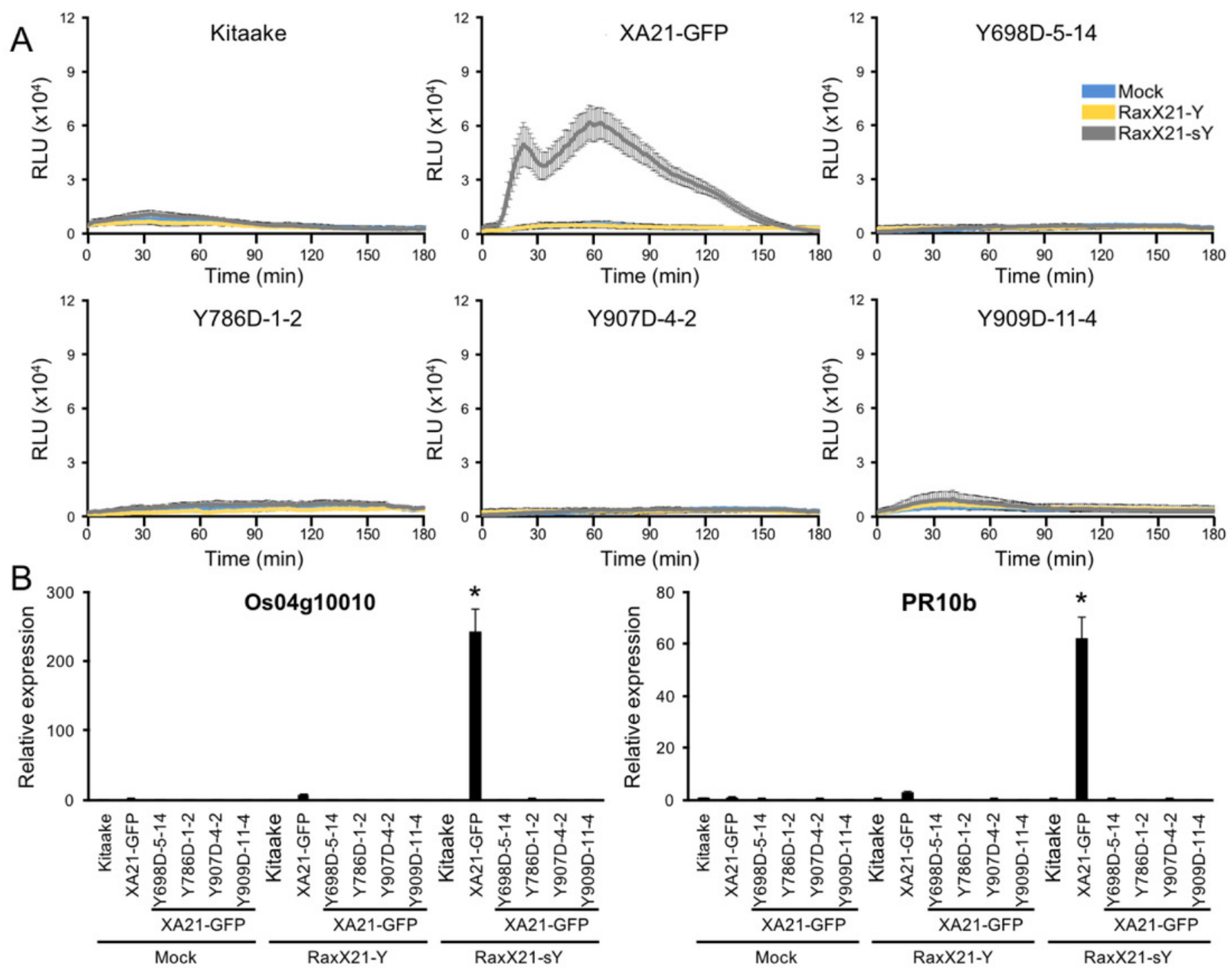


\section{Figure 6}

XA21 YF-GFP variants respond to sulfated RaxX.

(A) ROS production in rice leaves of Kitaake, XA21-GFP, and XA21 YF-GFP variants six hours after treatment with water (mock, blue), $1 \mu \mathrm{M}$ RaxX21-Y (yellow) or $1 \mu \mathrm{M}$ RaxX21-sY (gray). Data points depict the mean \pm standard error $(n=3)$. Each panel depicts a different plant background from a single experiment. RLU, relative light units. Note the Y-axis scale for Y698F-2-2 differs from the other plant lines. (B) Gene expression of Os04g10010 (left) and PR10b (Os12g36850, right) in rice leaves of Kitaake, XA21-GFP, and XA21 ${ }^{\mathrm{YF}}$-GFP variants siX hours after treatment with water (mock), $500 \mathrm{nM}$ RaxX21-Y, or $500 \mathrm{nM}$ RaxX21-sY. Bars depict the means \pm standard deviation $(n=3)$ of expression level normalized to mock-treated XA21-GFP. Different letters indicate a significant difference in gene expression $(P<0.05$, ANOVA, Tukey-HSD). The above experiments were performed twice with similar results. 

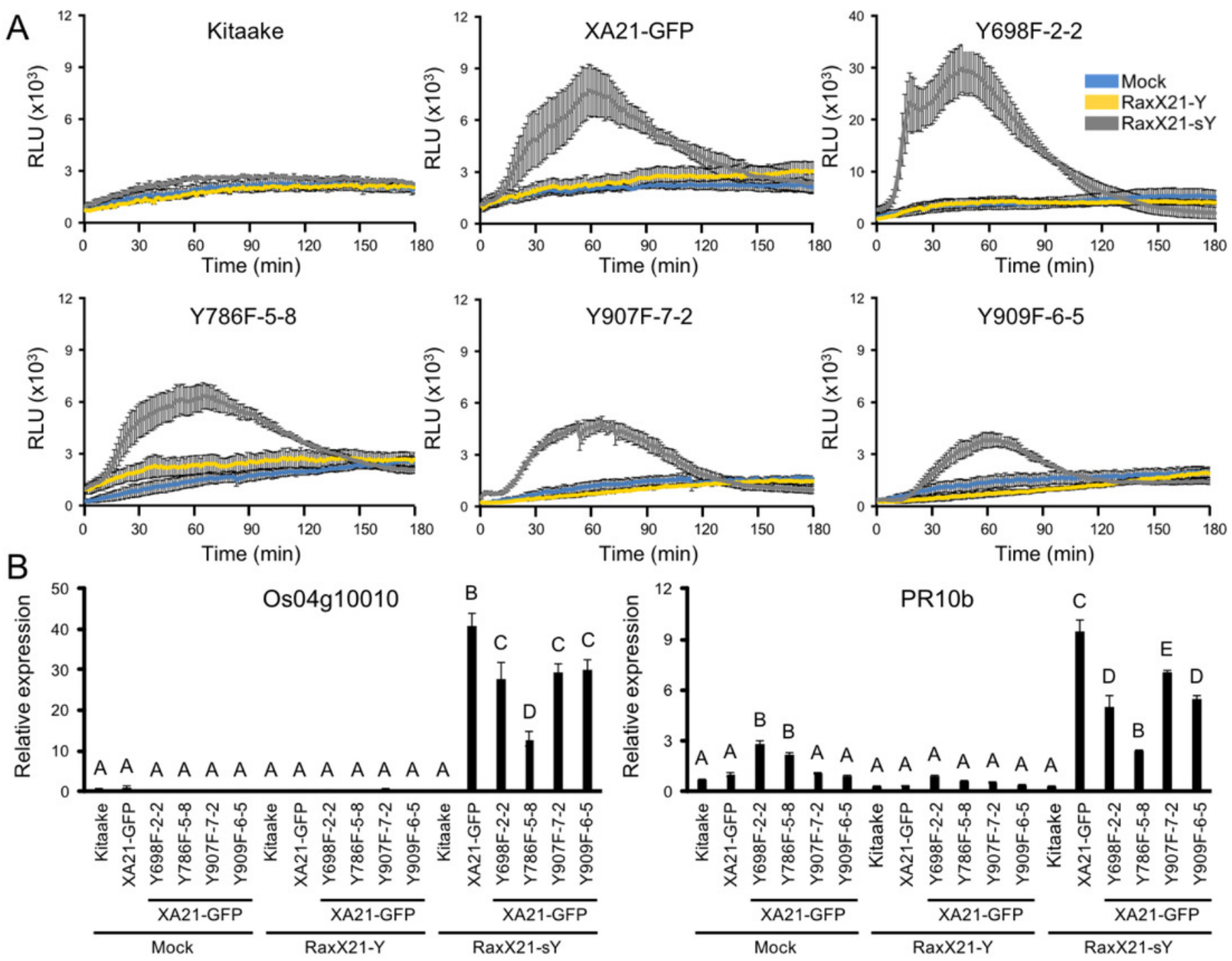


\section{Figure 7}

$X A 21 J K^{Y D}$ variants fail to interact with known XA21 binding proteins.

(A) The interaction between wildtype (WT) LexA-XA21JK and mutant variants (fused to the yeast GAL4 binding domain) and HA-tagged XB3, XB15, and juxtamembrane and kinase domains of OsSERK2 (OsSERK2JMK) (fused to the yeast GAL4 activation domain) were tested. Blue color indicates nuclear interaction between the two co-expressed proteins. (B) HA-XB3, HA-XB15, and HA-OsSERK2JMK fusion proteins are expressed in yeast. Anti-HA primary antibodies detected HA-fused XB3, XB15, or OsSERK2JMK protein (upper panel). The predicted sizes of HA-XB3, HA-XB15, and HA-OsSERK2JMK are $\sim 52 \mathrm{kDa}, \sim 80 \mathrm{kDa}$, and $\sim 65$ $\mathrm{kDa}$ respectively. Arrow indicates the band corresponding to the predicted size of HA-XB3. (C) WT LexA-XA21JK and mutant fusion proteins are expressed in yeast. Anti-LexA primary antibodies detected LexA-fused proteins (upper panel). LexA-GUS was used to confirm the absence of auto-activity in XB3, XB15, or OsSERK2JMK. The predicted size of LeXA-GUS is $\sim 110 \mathrm{kDa}$. The expected band size of LexA-XA21JK is $\sim 65 \mathrm{kDa}$. Equal loading of proteins in figure 7B and 7C was confirmed by Coomassie staining (CBB) the membrane after immunoblotting (lower panels). The above experiments were repeated twice with similar results. 


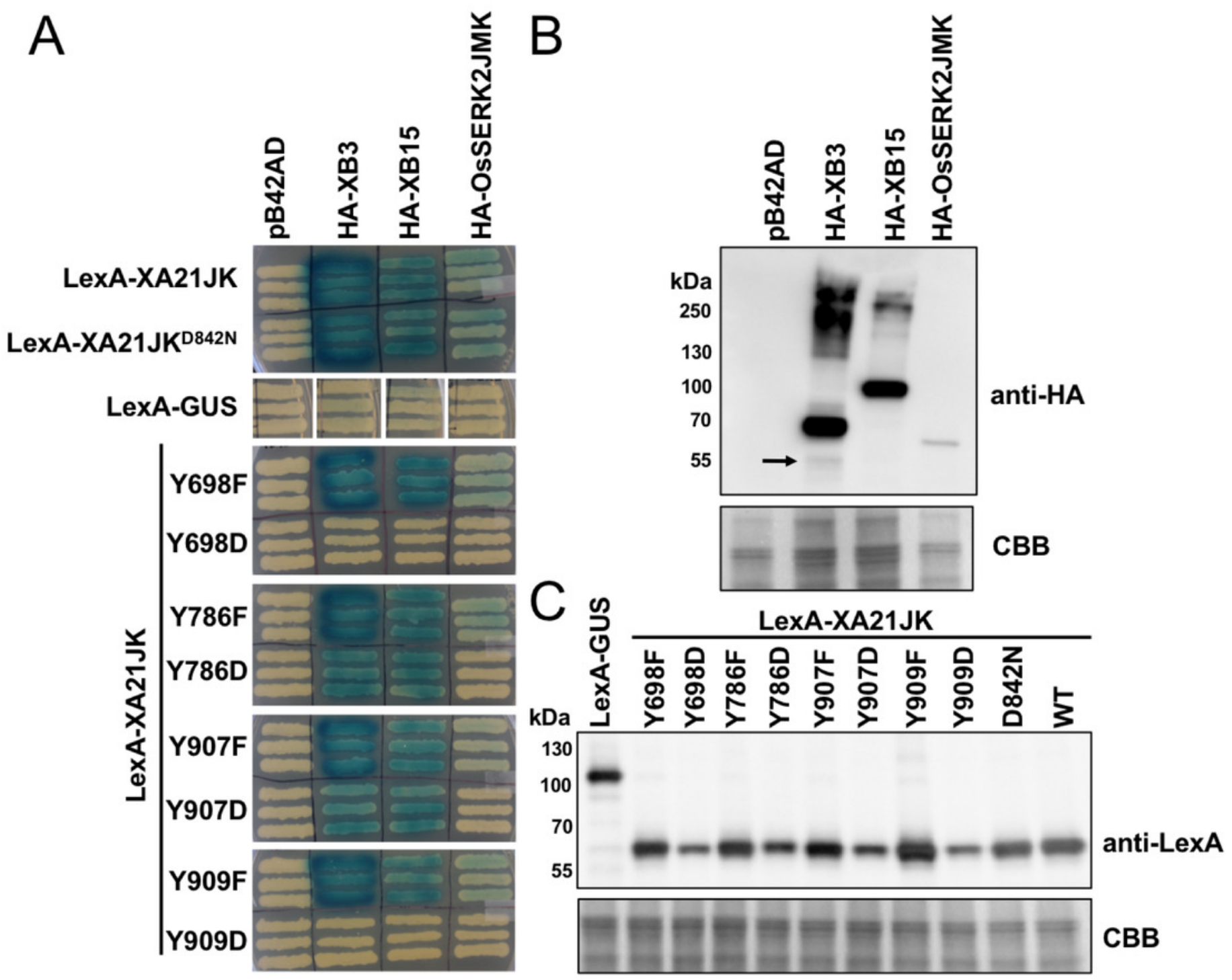

